\title{
Hybrid materials obtained by in situ polymerization based on polypropylene and mesoporous SBA-15 silica particles: catalytic aspects, crystalline details and mechanical behavior
}

\author{
Rosa Barranco-García ${ }^{1,2}$, Ana E. Ferreira ${ }^{3}$, M. Rosário Ribeiro ${ }^{*}$, Vicente Lorenzo ${ }^{4}$, Alberto García- \\ Peñas ${ }^{5,6}$, José M. Gómez-Elvira1 ${ }^{1}$ Ernesto Pérez¹, Maria L. Cerrada1*
}

\begin{abstract}
1'nstituto de Ciencia y Tecnología de Polímeros (ICTP-CSIC). Juan de la Cierva 3, 28006 Madrid, Spain
2PhD Program in Industrial Technology: Chemical, Environmental, Energetic, Electronic, Mechanical and Materials at Universidad Rey Juan Carlos, Madrid 28933, Spain.

${ }^{3}$ Centro de Química Estrutural, Instituto Superior Técnico, Universidade de Lisboa, Portugal

"Grupo de Investigación "POLímeros: Caracterización y Aplicaciones" (U. A. del ICTP-CSIC), E.T.S.I. Industriales, Universidad Politécnica de Madrid, José Gutiérrez Abascal 2, 28006 Madrid, Spain

${ }^{5}$ College of Materials Science and Engineering, Shenzhen Key Laboratory of Polymer Science and Technology, Guangdong Research Center for Interfacial Engineering of Functional Materials, Nanshan District Key Laboratory for Biopolymers and Safety Evaluation, Shenzhen University, Shenzhen 518060, P.R. China

6Key Laboratory of Optoelectronic Devices and Systems of Ministry of Education and Guangdong Province, College of Optoelectronic Engineering, Shenzhen University, Shenzhen 518060, P. R. China

*E-mail addresses of corresponding authors:
\end{abstract}

mlcerrada@ictp.csic.es (M. L. Cerrada); rosario@tecnico.ulisboa.pt (M.R. Ribeiro)

\section{Abstract}

Nanocomposites based on polypropylene have been prepared by in situ polymerization of propene in presence of mesoporous SBA-15 silica. Synthetic reactions were carried out with a zirconocene catalyst under either homogenous or supported conditions, the SBA-15 particles being used as carriers, and testing the immobilization of the catalytic system by several approaches. The existence of polypropylene able to crystallize within the mesoporous channels in the resulting materials is initially figured out from the appearance of a small endothermic process on heating calorimetric experiments, located at around $100{ }^{\circ} \mathrm{C}$. The presence of polypropylene crystallites confined within the SBA-15 mesostructure is thereafter confirmed by SAXS measurements through the intensity variation of the SBA-15 first order reflection, this change being dependent on composition. Accordingly, polypropylene chains can grow up either outside or inside the SBA-15 channels during polymerization and the mesoporous particles maintain their ordered hexagonal arrangement. Mechanical response, as deduced from indentation measurements, improves with SBA-15 incorporation (without varying the final processing temperature). Thus, stiffness increases and deformability is reduced in the nanocomposites as the SBA-15 content rises. Simultaneously, polypropylene amount within channels is enlarged. Amount of SBA-15 is, then, the most important variable.

Keywords: Polypropylene; mesoporous SBA-15; endothermic process; SAXS; indentation modulus. 


\section{Introduction}

Isotactic polypropylene, iPP, is characterized by its relatively low manufacturing cost and its rather interesting properties, turning out as one of most versatile polymers actually produced at industrial level [1-5], both as a plastic and as a fiber. The use of metallocene catalysts constituted a great tool from mid 90s to synthesize under homogenous conditions polypropylenes with low amounts of oligomers and different tacticities [6] (atactic, isotactic or isoblock, among others), and also copolymers with ethylene, 1-butene and longer chain a-olefins [7-12] with high comonomeric molar fractions. Consequently, properties could be tunable depending on the ultimate requirements. Thus, the main attribute of those metallocene catalysts was their amazingly large variety and vast versatility since, besides polypropylene, several classes of polyolefins (homo- and copolymers by polymerizing cycloolefin or dienes, for instance) were available, having been inaccessible so far by any other catalytic system and polymerization process. However, metallocene complexes as homogeneous catalysts can work out industrially only in solution process and they must be heterogenized for slurry and gas phase reactors. Their immobilization, if properly performed, should not damage their performance and should lead to supported catalyst that could produce polymer particles with controlled morphology. The initial attempts of their heterogenization were performed by using silica and alumina as inorganic supports [13-15]. Zeolites [16] were later tried. Carbon nanostructures (nanotubes, graphite, graphene oxide, among others) have been also used as supports to obtain polypropylene based materials by in situ polymerization with either Ziegler-Natta or metallocene catalysts [17-20].

This interest in the immobilized metallocene catalysis is currently focused in the implementation of new carriers and impregnation techniques. The most commonly used substrates have been silicabased materials. Mesoporous silicas, such as MCM-41 [21] and SBA-15 [22], have received recently a great attention. These mesostructured silicas exhibit regular pores with diameters ranging from around 2 to $20 \mathrm{~nm}$. They may act as polymerization nanoreactors, affecting the pattern of monomer insertion and, consequently, the polymer morphology. Research articles demonstrated the ability of these mesoporous silicas to produce nanometer scaled PE through space confined polymerization [23-25]. Dong et al. produced PE nanofibers with $\mathrm{Cp}_{2} \mathrm{ZrCl} 2$ fixed on $\mathrm{MCM}-41$ and $\mathrm{SBA}-15$ where the later tubular structures possess larger diameters than the former [26]. Xu et al. [27] obtained, after supporting an iron(II)bisimine pyridine catalyst on SBA-15, polyethylene with fibrous morphology and higher molar mass than its homogeneous powdered counterpart. Formation of PE nanofibers was also described by Campos et al. [28] when using zirconocene supported on MCM-41 and Al containing MCM-41 carriers, this fibrous morphology being favored at low polymerization activities. 
The use of nanostructured silica supports in olefin polymerization and its effect on catalytic activity, morphologies and physical properties of polyolefins showed their role and importance in the preparation of novel polyolefin nanoblends and nanocomposites [25]. Special interactions between MCM-41 and polyethylene were reported [29]. Those resulting self-reinforced HDPE/MCM-41 nanocomposites revealed an improved mechanical performance and an easier degradability at the end of the life cycle $[24,29]$. Efforts for enhancing the dispersion of mesoporous MCM-41 particles (either micro or nanosized) within HDPE matrix by in situ polymerization were described later on. They involved either the functionalization of HDPE chains by copolymerization [30] with a polar monomer or the modification of MCM-41 surface by several silanes before polymerization [31]. Moreover, in situ polymerization has been just proved as useful approach to synthesize nanocomposites based on ultrahigh molecular weight polyethylene (UHMWPE) and SBA-15 particles [32] and UHMWPE/HDPE inreactor blends [33].

Studies concerning in situ polymerization of propylene on commercial silica using metallocene catalysts can be also found in literature. A strategy involved the pretreatment of zirconocene with methylaluminoxane (MAO) before the catalyst impregnation and an increase in the molecular weight was observed [34]. Other studies showed that the amount of catalyst that can be immobilized increased by pretreating the support with MAO [35], leading to a better catalytic activity during polymerization. Improvements in this parameter were also observed by substituting microsized MCM-41 for the corresponding MCM-41 nanoparticles [36] because of reduction of constraints to mass transfer. Nanocomposites of PP and MCM-41 nanoparticles achieved by in situ polymerization have been found once in literature [37], although properties of those resulting materials were not described.

The present work aims the obtainment of (nano)composites based on isotactic polypropylene and mesoporous SBA-15 particles by in situ polymerization using rac-ethylenebis(indenyl)zirconium (IV) dichloride as a metallocene catalyst and MAO as cocatalyst. Three different impregnation approaches have been checked to immobilize this metallocene on the SBA-15 carrier. Their effect on the polymerization activity has been investigated and compared with the one exhibited by polypropylene attained under homogeneous conditions. Moreover, the SBA-15 content in the final material has been assessed by thermogravimetric analysis. The most interesting nanocomposites have been more deeply evaluated. Thus, their crystalline structure has been analyzed by X-ray diffraction as well as the phase transitions associated with these ordered entities by differential scanning calorimetry. The ordered structure of the SBA-15 particles in the distinct materials has been checked by small angle X-ray scattering. And, finally, the ultimate mechanical performance has been tested by indentation experiments. 


\section{Experimental part}

\section{Materials and chemicals}

The SBA-15 particles were purchased from Sigma-Aldrich (specific surface area, $\mathrm{S}_{\mathrm{BET}}=517$ $\mathrm{m}^{2} / \mathrm{g}$; total pore volume, $V_{t}=0.83 \mathrm{~cm}^{3} / \mathrm{g}$; average mesopore diameter [38], $D_{p}=6.25 \mathrm{~nm}$ ). Prior its use, SBA-15 was dried under a flux of dry air $(7.6 \mathrm{~L} / \mathrm{g} . \mathrm{h})$ at $300{ }^{\circ} \mathrm{C}$ for $1 \mathrm{~h}$. The temperature was increased from 20 to $300{ }^{\circ} \mathrm{C}$ at $5^{\circ} \mathrm{C} / \mathrm{min}$. Then, the support was kept at this temperature during another $1 \mathrm{~h}$ under a nitrogen flow $(7.6 \mathrm{~L} / \mathrm{g} . \mathrm{h})$ and finally cooled down to room temperature and stored under dry nitrogen in a Schlenk flask.

All experiments carried out for the SBA-15 modification and propylene polymerization were performed under dry nitrogen using standard Schlenk techniques. Propylene and nitrogen (Air Liquide) were purified through absorption columns containing molecular sieves $4 \mathrm{~A}$ and 13X. Racethylenebis(indenyl)zirconium (IV) dichloride (rac-Et[Ind $]_{2} \mathrm{ZrCl}_{2}$ ) and methylaluminoxane (MAO, 7 wt. \% Al in toluene solution, AkzoNobel) were also used as received. Toluene (VWR Chemicals) was dried by refluxing over metallic sodium under a dry nitrogen atmosphere, using benzophenone as indicator.

\section{Propylene polymerizations}

Polymerizations were performed in a $250 \mathrm{~mL}$ dried and nitrogen-flushed bottle for pressure reactions (Wilmad LabGlass LG-3921), magnetically stirred. The reactor was filled with toluene $(50 \mathrm{~mL})$ and the adequate amount of the MAO co-catalyst, the catalyst and propylene. Polymerizations were carried out at $25^{\circ} \mathrm{C}$ and at a pressure of $1.2 \mathrm{bar}$ in propylene. The polymerization was monitored and ran until a given amount of propylene was consumed, allowing the control in SBA-15 content for the diverse PP nanocomposites when a catalyst immobilization protocol was used. Different reaction times, as parameter of control, were used for the polymerizations under either homogeneous or supported conditions. Polymerizations were quenched by the addition of methanol acidified with $10 \% \mathrm{HCl}$. The polymer was, then, collected and washed twice with methanol before drying.

Reaction parameters, as temperature, pressure and propylene consumption, were monitored in real time and data stored, enabling achievement of kinetic profiles. Propylene consumption rate was measured using two mass flow controllers (Hastings Instruments HFC-202 and Alicat Scientific 16 Series) and recorded in a computer (Computer Boards CIO-DAS08/Jr-A0 interface card with Labtech DataLab software). The propylene mass flow had the units of SLPM (standard liter per minute) and was converted to propylene consumption with the units $\mathrm{kg} \mathrm{PP} /(\mathrm{mol} \mathrm{Zr} . \mathrm{h})$. Kinetic profiles corresponded to 
propylene consumption data versus time while integral of the obtained curve as function of time was ascribed to average value of the activity. Results were in agreement with weight of the attained polymer.

\section{Preparation of the supported catalyst}

Three different methods were used for the immobilization of catalyst on the mesoporous SBA15 carrier as detailed below:

Direct impregnation (DI method)

SBA-15 $(0.10 \mathrm{~g})$ was contacted with the zirconocene catalyst $\left(1.9 \times 10^{-6} \mathrm{~mol}\right)$, in the form of a solution $1.5 \times 10^{-3} \mathrm{M}$ in toluene, for a given period of time, in a Schlenk flask tube under magnetic stirring, concealed from ambient light. In order to confirm that all the zirconocene was immobilized on the mesoporous particles, the suspension containing the catalytic system, obtained after the contact time between the mesoporous carrier and the catalyst, was allowed decanting. Then a small volume ( $2 \mathrm{~mL}$ ) of the clear supernatant liquid was tested in polymerization conditions, with further addition of MAO (the same used for polymerization runs). The polymerization test with this clarified solution did not lead to any activity, confirming that no catalyst remained in the supernatant solvent [32].

Pretreatment of SBA-15 with MAO and impregnation of the zirconocene on pretreated support (SBAMAO method)

SBA-15 was first contacted with MAO in a Schlenk flask under nitrogen atmosphere at room temperature by addition of $1.35 \mathrm{~mL}$ of MAO to $1 \mathrm{~g}$ of support dispersed in $25 \mathrm{~mL}$ of toluene. After $16 \mathrm{~h}$ under stirring, the solid was washed three times with ca. $20 \mathrm{~mL}$ of dry toluene and dried at room temperature under vacuum overnight.

Once the MAO treated SBA-15 compound was completely dried, $100 \mathrm{mg}$ of this solid was contacted with $1.9 \mu \mathrm{mol}$ of zirconium catalyst in toluene and stirred for $10 \mathrm{~min}$. After this period of time, the catalyst suspension underwent the clarified liquid test described above. Since the test was successful, a new catalyst suspension was prepared at the same conditions for being used in the propylene polymerizations.

Impregnation of pre-activated Zr catalyst with MAO on SBA-15 (PA method)

A solution of the zirconocene in toluene was pre-activated with MAO by stirring for $15 \mathrm{~min}$ at room temperature. Two Al/Zr ratios were tested: 150 and 300, as seen in Table 1. After this time, the equivalent of $1.9 \mu \mathrm{mol}$ of MAO pre-activated catalyst was mixed with $100 \mathrm{mg}$ of the support in toluene 
and stirred for 10 min. As in DI or SBA-MAO approaches, no activity was observed for the supernatant liquid after immobilization when further tested in polymerization conditions. Thus, catalyst was not remaining in the homogeneous solution.

\section{Characterization of homopolymers and nanocomposites}

\section{Size exclusion chromatography}

Molecular weights were evaluated for some of the synthesized materials by size exclusion chromatography (SEC) at $145^{\circ} \mathrm{C}$ in a Waters GPC/V 2000 equipment with both refractive index and viscosimeter detectors. A set of three columns of the PL Gel type was used with 1,2,4-trichlorobenzene as solvent,and a flow rate of $1 \mathrm{~mL} / \mathrm{min}$. The analyses were calibrated with polystyrene standards of narrow molecular mass distributions. The molecular weights and polydispersity index $\left(M_{w} / M_{n}\right)$ are listed in Table 1.

\section{Nuclear Magnetic Resonance}

Tacticity was determined by carbon nuclear magnetic resonance analysis, ${ }^{13} \mathrm{C} N \mathrm{NR}$, from a polymeric solution in 1,1,2,2-tetrachloroethane- $d_{4}(70 \mathrm{mg} / 1 \mathrm{~mL})$ at $100{ }^{\circ} \mathrm{C}$, using a Bruker Avance III/500 spectrometer operating at $125.76 \mathrm{MHz}$. A minimum of 4000 scans were recorded with broad band proton decoupling, using an acquisition time of $1.3 \mathrm{~s}$ and a pulse delay of $5 \mathrm{~s}$.

\section{Preparation of films}

Powders obtained after polymerization were processed as films by compression molding in a Collin P-200-P press between hot plates at $190{ }^{\circ} \mathrm{C}$ for $3 \mathrm{~min}$ without pressure and $3 \mathrm{~min}$ at a pressure of 30 bar. A further fast cooling process was applied with cold water for 3 min at 30 bar. These films have been named as followed: iPPHOMx for the iPP homopolymers synthesized under homogeneous conditions, the $x$ being the used amount in $\mu$ mol of catalyst ( 5 and 1.9); and, iPPSBAy for the iPP composites containing mesoporous SBA-15, the $y$ being the average SBA- 15 wt. $\%$ content estimated by TGA.

\section{Thermogravimetric Analysis}

Dynamic thermal gravimetric experiments (TGA) were performed from $40{ }^{\circ} \mathrm{C}$ up to $700{ }^{\circ} \mathrm{C}$ in a Q500 equipment of TA Instruments under air or nitrogen atmosphere at a heating rate of $10{ }^{\circ} \mathrm{C} / \mathrm{min}$. Samples were discs cut from the film with a die of $4 \mathrm{~mm}$ diameter, approximately, $4 \mathrm{mg}$. These 
measurements were carried out for assessment of final SBA-15 content in a given nanocomposite as well as for evaluation of the degradation processes of the resulting materials.

X-ray experiments with conventional and synchrotron radiation

Wide angle X-Ray Diffraction (WAXD) patterns at room temperature were recorded to examine the crystalline structure of the polymeric matrix in the reflection mode by using a Bruker D8 Advance diffractometer provided with a PSD Vantec detector (from Bruker, Madison, Wisconsin). Cu Ka radiation $(\lambda=0.15418 \mathrm{~nm})$ was used, operating at $40 \mathrm{kV}$ and $40 \mathrm{~mA}$. The parallel beam optics was adjusted by a parabolic Göbel mirror with horizontal grazing incidence Soller slit of $0.12^{\circ}$ and LiF monochromator. The equipment was calibrated with different standards. A step scanning mode was employed for the detector. The diffraction scans were collected with a $2 \theta$ step of $0.024^{\circ}$ and $0.2 \mathrm{~s}$ per step.

Real-time variable-temperature simultaneous SAXS/WAXD experiments were carried out with synchrotron radiation in beamline BL11-NCD at ALBA (Cerdanyola del Valles, Barcelona, Spain) at a fixed wavelength of $0.1 \mathrm{~nm}$ to mainly analyze the hexagonal ordered arrangement of the mesoporous SBA-15 silica. An ADSC detector has been used for SAXS (off beam, at a distance of $292 \mathrm{~cm}$ from sample) and a Rayonix one for WAXD (at about $14 \mathrm{~cm}$ from sample, and a tilt angle of around 30 degrees). A Linkam Unit was employed for the temperature control. The calibration of spacings was obtained by means of silver behenate and $\mathrm{Cr}_{2} \mathrm{O}_{3}$ standards. The initial $2 \mathrm{D}$ X-ray images were converted into 1D diffractograms, as function of the inverse scattering vector, $s=1 / d=2 \sin \theta / \lambda$. Film samples of around $5 \times 5 \times 0.1 \mathrm{~mm}$ were employed in the synchrotron analysis.

\section{Differential Scanning Calorimetry Analysis}

Calorimetric analyses were carried out in a TA Instruments Q100 calorimeter connected to a cooling system and calibrated with different standards. The sample weights were around $3 \mathrm{mg} . \mathrm{A}$ temperature interval from -40 to $180{ }^{\circ} \mathrm{C}$ was studied at a heating rate of $20{ }^{\circ} \mathrm{C} / \mathrm{min}$. For the determination of the crystallinity, a value of $160 \mathrm{~J} / \mathrm{g}$ was used as the enthalpy of fusion of a perfectly crystalline material [39-41].

\section{Scanning electron microscopy}

Scanning electron microscopy, SEM, was carried out at room temperature in XL30 ESEM PHILIPS equipment for a dispersion of SBA-15 particles in acetone and for some of the synthesized materials. These specimens were deposited in a holder prior to observation. 
Depth Sensing Indentation, DSI, experiments were performed at $20^{\circ} \mathrm{C}$ with a Shimadzu tester (model DUH211S) equipped with a Berkovich-type diamond indenter. At least 10 distinct indentations were carried out at different regions on the surface for the distinct materials analyzed, isotactic polypropylene homopolymer and nanocomposites based on isotactic polypropylene and SBA-15 particles. The experimental protocol consisted in several stages: a) application of a load of $10 \mathrm{mN}$ at a loading speed of $1.46 \mathrm{mN} / \mathrm{s}$; b) maintenance of this constant load for $5 \mathrm{~s}$; and c) release of the load at an unloading speed equal than the one used along the loading stage. Finally, indentation depth was registered for 5 additional seconds after reaching the minimum load $(0.1 \mathrm{mN})$.

Martens hardness, $\mathrm{HMs}$, as well as indentation hardness, $H_{i t}$, and modulus, $E_{i t}$, were calculated according to Oliver-Pharr method [42]. These two hardness values are given by the ratio of the maximum load to either the contact area under load or after releasing the indentor, respectively. Consequently, $\mathrm{HMs}$ is related to elastic, viscoelastic and permanent strains, whereas $\mathrm{H}_{\text {it }}$ only depends on viscoelastic and plastic strains.

\section{Results and discussion}

\section{Propylene polymerizations and morphological characteristics}

Polymerizations under homogeneous conditions were first investigated by the selection of a suitable protocol to be applied later during the reactions with the zirconocene catalyst immobilized onto mesoporous SBA-15 through the three different mentioned approaches. The effect of experimental parameters, and methodology was tested and the obtained results are summarized in Table 1. These preliminary polymerization tests were conducted with the homogeneous catalysts at different catalyst concentrations and the kinetic profiles are represented in Figure S1 of Supporting Information. The homopolymers display a low molar mass (see values in Table 1) independently of the synthetic parameters. The selected rac-Et[lnd $]_{2} \mathrm{ZrCl}_{2}$ zirconocene catalyst has a primary role in this feature, since its use led to polypropylenes of relatively low molar mass and isotacticity (around 86-89 \%) [43]. The ${ }^{13} \mathrm{C}$ NMR results show that a value of $89 \%$ is achieved for the homopolymers under the experimental conditions explored (see iPPHOM1.9PA spectrum in Figure S2 of Supporting Information). As positive aspect, this catalyst is less sensitive to the presence of impurities during the reaction process, this characteristic being very convenient for the fine tuning of any synthetic protocol. 
Table 1. Polymerization conditions and activities for the synthesis of PP/SBA nanocomposites performed under homogeneous and supported polymerizations.

\begin{tabular}{|c|c|c|c|c|c|c|}
\hline Sample & Method & $\mathrm{Al} / \mathrm{Zr}$ & $\begin{array}{c}\text { Average } \\
\text { activity } \\
\text { (kgPP/molZr.h) }\end{array}$ & $\begin{array}{c}\mathrm{M}_{\mathrm{w}} \\
(\mathrm{g} / \mathrm{mol})\end{array}$ & $\mathrm{M}_{w} / \mathrm{M}_{\mathrm{n}}$ & $\begin{array}{l}\text { SBA-15 } \\
\text { (wt.\%) }\end{array}$ \\
\hline \multicolumn{7}{|c|}{ HOMOGENEOUS POLYMERIZATIONS } \\
\hline iPPHOM5 & - & 500 & $3050^{*}$ & - & - & 0 \\
\hline iPPHOM1.9 & - & 500 & 2100 & 42000 & 2.2 & 0 \\
\hline iPPHOM1.9PA & PA-150 & 650 & 2200 & 43500 & 2.2 & 0 \\
\hline \multicolumn{7}{|c|}{ SUPPORTED POLYMERIZATIONSa } \\
\hline & DI & 650 & 175 & - & - & 42 \\
\hline iPPSBA59 & DI & & & - & - & 59 \\
\hline iPPSBA10 & PA-150 & & & 51270 & 2.5 & 10 \\
\hline iPPSBA21 & PA-150 & 650 & 930 & 50270 & 4.1 & 21 \\
\hline iPPSBA57 & PA-150 & & & - & - & 57 \\
\hline iPPSBA9 & PA-300 & & & 46900 & 2.6 & 9 \\
\hline iPPSBA13 & PA-300 & 800 & 820 & - & - & 13 \\
\hline iPPSBA18 & PA-300 & & & 50250 & 2.6 & 18 \\
\hline iPPSBA15 & SBA-MAO & & & - & - & 15 \\
\hline iPPSBA16 & SBA-MAO & 650 & 550 & 52290 & 2.6 & 16 \\
\hline iPPSBA20 & SBA-MAO & & & - & - & 20 \\
\hline
\end{tabular}

a Propylene pressure $=1.2 \mathrm{bar}, \mathrm{T}=25^{\circ} \mathrm{C}$; catalyst loaded in the support $=1.9 \mu \mathrm{mol} / \mathrm{g} ;$ Impregnation time $=10$ $\min$.

${ }^{*}$ Activity assessed at 30 min with the exception of one remarked specimen where estimation was at 12 min.

Figure $\mathrm{S} 1$ shows the effect that the amount of catalyst ( 5 or $1.9 \mu \mathrm{mol})$ at constant $\mathrm{Al} / \mathrm{Zr}$ ratio exerts in the instantaneous activity. Results are found rather analogous between PPHOM5 and iPPHOM1.9. Thus, $1.9 \mu \mathrm{mol}$ in rac-Et[lnd $]_{2} \mathrm{ZrCl}_{2}$ is selected for cost-effective and environmental reasons. A synthetic modification was introduced, sample labeled as iPPHOM1.9PA, since the activities reached under those homogenous conditions were not very high at this polymerization temperature. That consisted of a previous pre-contact of metallocene with the MAO co-catalyst before polymerization starts $[34,35]$. Its effect is very significant, as seen in Figure S1. This pre-activation step leads to an 
important rise of the instantaneous activity at the beginning of reaction compared with the behavior observed in the other homopolymers. This feature agrees with the behavior described for the ethylene polymerization [35]. Figure S1 also depicts clearly that deactivation of the catalytic system starts, however, at shortest times and more severely. Accordingly, the productivity at $30 \mathrm{~min}$ is rather analogous in both nanocomposites IPPHOM1.9 and iPPHOM1.9PA. These results cannot be referred to previous ones in the literature since this catalyst has been immobilized onto mesoporous SBA-15, to the best of our knowledge, for the first time to obtain polypropylene based derivatives. There is a preceding work involving propylene and mesoporous silica, but in that case, MCM-41 [37] was used instead of SBA-15 and nano instead of microsized particles were employed. Moreover, the chosen zirconocene was $\mathrm{Me}_{2} \mathrm{Si}(\mathrm{Ind})_{2} \mathrm{ZrCl}_{2}$, which usually leads to higher molar mass and isotacticity [37]. These authors observed a rise in the activity and a reduction of molar mass with increasing temperature.

The polymerization behavior for the supported catalyst using three different impregnation strategies is summarized in Table 1 and depicted in Figure 1. Different SBA-15 compositions are achieved by changing polymerization time at a specific approach. The polymerization runs carried out at 30 min were used to calculate the polymerization activity and are indicative of the typical values obtained for each one of the used methodologies. As expected, by immobilization of the catalyst rac$E t[I n d]_{2} \mathrm{ZrCl}_{2}$ the molar mass is increased. On the other hand, propylene tacticity, which was estimated in the resultant composites from the methyl region at triad level, remains rather unchanged at about $89 \%$, independently of the impregnation strategy used. This value is similar to that found in the homopolymer (see Figure S2). Moreover, a loss in the activity is observed compared with those magnitudes found in their homogeneous homologous. Reduction is slightly above $40 \%$ in the most favorable reactions. It is also deduced that the less efficient approach is the DI. A very strong deactivation takes place at very short times and the activity values become extremely low. Accordingly, very high SBA-15 contents (determined by TGA and commented later on) are present in the final achieved materials. The SBA-MAO methodology increases significantly the activity and reduces the deactivation in relation to the $\mathrm{DI}$ approach, as seen in Figure 1. The PA strategy turns out the method leading to the best results in polymerization activity. It is noticed that there are not important differences between the results obtained for the two AI/Zr ratios tested, PA150 and PA300. The aforementioned reduction in the activity can be related to the deactivation pathways that might occur during immobilization. Then, not all of the supported catalyst on the surface of the mesoporous SBA-15 is in an active form to react with the propylene. Diffusional effects operating in the SBA-15 particles might also contribute to the decrease of polymerization activity within the heterogeneous systems [31]. 


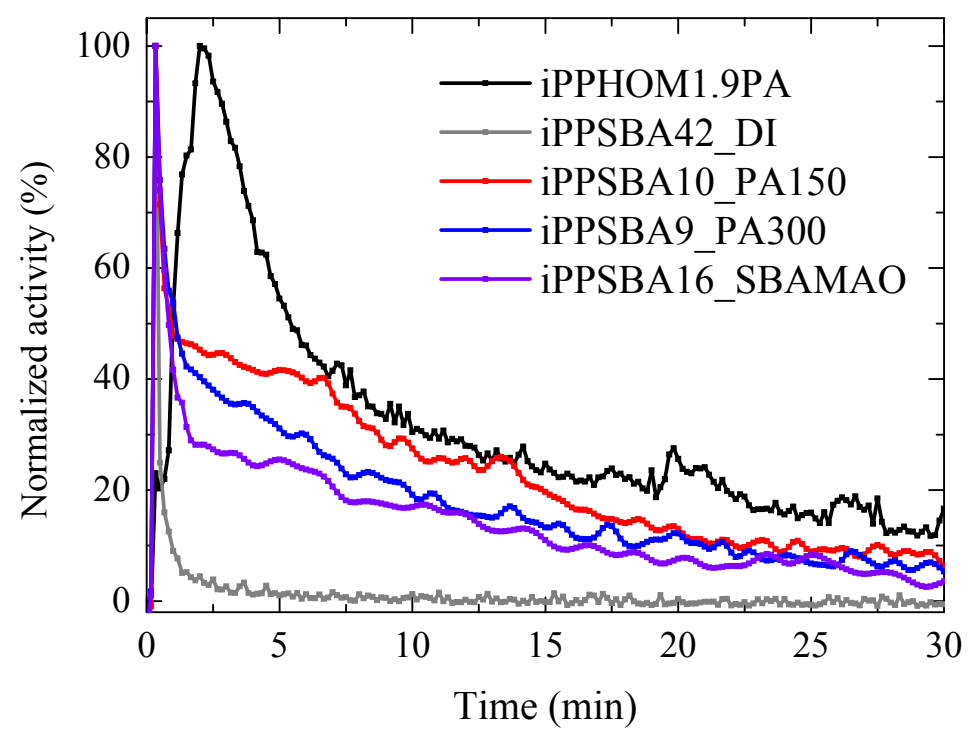

Figure 1. Normalized kinetic profiles for the propylene polymerizations carried out by distinct immobilization approaches compared with the homopolymer attained under homogeneous conditions.

Figure S3 of Supporting Information displays that SBA-15 particles are rather uniform in size and shape, as figured out from picture a). They present their characteristic vermicular elongated shape with an average size of $350 \mathrm{~nm}$ wide and $0.9 \mu \mathrm{m}$ long. On the other hand, the different hybrid materials exhibit a homogeneous dispersion of the mesoporous silica particles within the polypropylene matrix. Its polymerization from surface of the catalyst carrier seems to have hampered the SBA-15 particle agglomeration and boosted the fine filler distribution. It is clearly noticeable, as deduced from Figure S3 b) and c), the higher amount of the inorganic component existing in the IPPSBA18 specimen compared with that in the iPPSBA9 sample because of their differences in SBA-15 content. A higher magnification of the former material (micrograph d) in Figure S3) points out that in situ polymerization has led to a suitable filler-matrix interfacial adhesion and an intimate contact between the SBA-15 and polypropylene. Thus, particles are completely embedded within the matrix and detectable inorganic domains are absent across the surface.

\section{Thermogravimetric analysis}

Once the different IPP based materials, either neat homopolymers or those incorporating SBA15, have been synthesized through different methodologies, knowledge of some of their physical properties is required. The mesoporous SBA-15 used as catalyst carrier during the preparation was not removed at the end of this synthetic procedure. Since polymerization is expected to occur both in the 
external surface and within the nanometric mesopores, the resulting materials can be considered nanocomposites comprising a polymeric matrix and the mesoporous SBA-15 particles as filler. Determination of SBA-15 amount is mandatory because the important role that exerts in the ultimate response. Thermogravimetric analysis constitutes an easy tool to learn about the SBA-15 content as well as about the thermal stability and presented decomposition stages. To attain reliable average values in the resultant materials, experiments have been performed from either the initial polymerization powders or from the films obtained after their compression molding, each one under two distinct atmospheres (inert and oxidative). Then, the average final SBA-15 content listed in Tables 1 and 2 for a given specimen is the average result achieved from these four different measurements. Rather analogous SBA-15 values are attained, pointing out the fairly homogeneity exhibited by these in situ polymerized materials. This correlated well with the morphological aspects commented above.

Figures 2 and 3 show the TGA curves under inert and oxidative conditions, respectively, for some of the materials prepared by the different immobilization approaches. Figure 2 seems to point out that all of the specimens display a single primary stage of decomposition under an inert atmosphere in the temperature range from 300 to $500{ }^{\circ} \mathrm{C}$. The reaction products of polypropylene pyrolysis consist of four major categories [44]: alkanes, alkenes, dienes, and aromatic compounds. The two former ones are present in significantly greater yields than the latter two compounds. Most of the alkenes appear in the form of $C_{3 n}$, the alkanes in the form of $C_{3 n-1}$, and the dienes in the form of $C_{3 n-2}$. This product distribution is explained by the typical free-radical mechanism involving bond fission, hydrogen abstraction, $\beta$-scission, intramolecular hydrogen transfer, and radical recombination as the dominant steps.

TGA curves of these composites under air exhibit, at least, two degradation processes at similar temperature interval, as depicted in Figure 3. It is well known that the initial reaction of polyolefins during thermal oxidation is the formation of alkyl radicals from polymeric chains followed by the reaction of alkyl radicals with oxygen to form hydroperoxides [45,46], which can decompose to alkoxyl radicals. Then, the alkoxyl radicals abstract hydrogen from the chain and other alkyl radical forms. Finally, various carbonyl species are generated.

The incorporation of SBA-15 particles does not apparently appear to affect the thermal stability of the different materials either under inert or air conditions, with the exception of the iPPSBA57 in an air environment, in which the process involving the largest weight loss is shifted to higher temperature. The effect of mesoporous particles in the thermal degradation of in situ polymerized polypropylene is not reported in literature. For polyethylene, their greater or smaller influence is rather dependent on the type 
of polyethylene (high density polyethylene, HDPE, compared with ultra-high molecular weight polyethylene, UHMWPE) and on using pristine or decorated mesoporous particles. An easier degradability [24] was usually found under inert conditions in materials containing HDPE and neat mesoporous MCM-41 and in those incorporating either MCM-41 modified with UA/TIBA (undecenoic acid/triisobutylaluminum) [47] or MCM-41 decorated with different silanes [48]. A shift to slightly inferior temperatures was also observed in materials based on UHMWPE and on polyethylene synthesized with a hafnocene catalyst, both containing SBA-15 particles $[32,49]$.
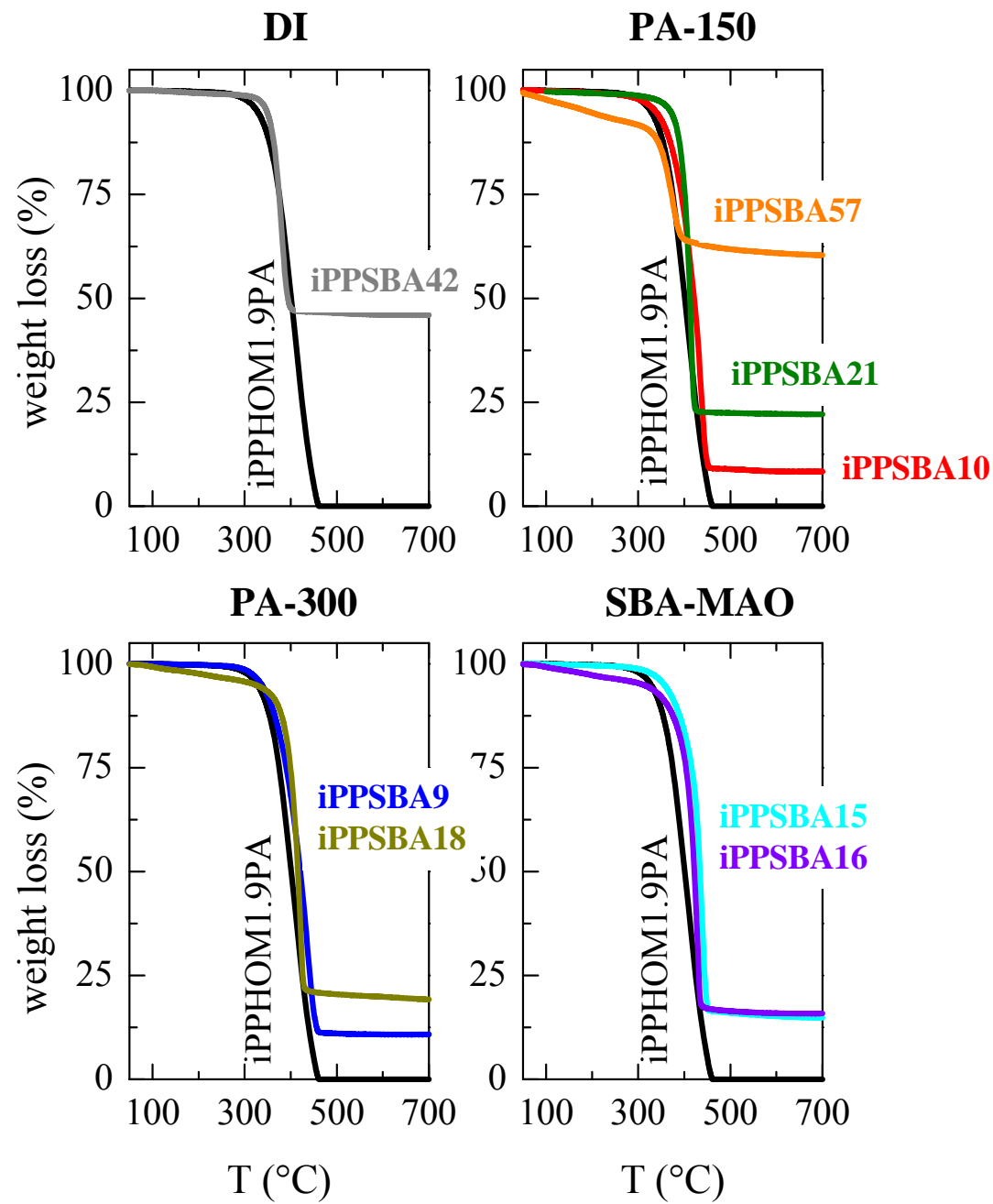

Figure 2. TGA curves under inert atmosphere for the neat iPPHOM1.9PA (taken as reference) and its nanocomposites prepared by different catalyst impregnation approaches.

It should be noted that the weight loss actually begins around $100{ }^{\circ} \mathrm{C}$, regardless of the atmosphere (inert or oxidant) in which the experiment is performed. This initial loss is related to the water adsorbed by the composites and the adsorption comes from the contact with the ambient water 
vapor because of the hydrophilic nature of the silica SBA-15. Therefore, the greatest loss is observed in the iPPSBA57 that contains the highest amount of SBA-15. A drying process was not applied to remove any physically adsorbed water prior to these TGA experiments due to the interest of learning the pristine thermal behavior.

DI

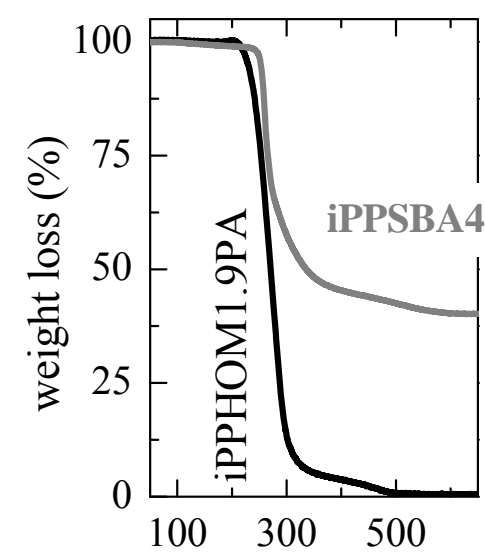

PA-300
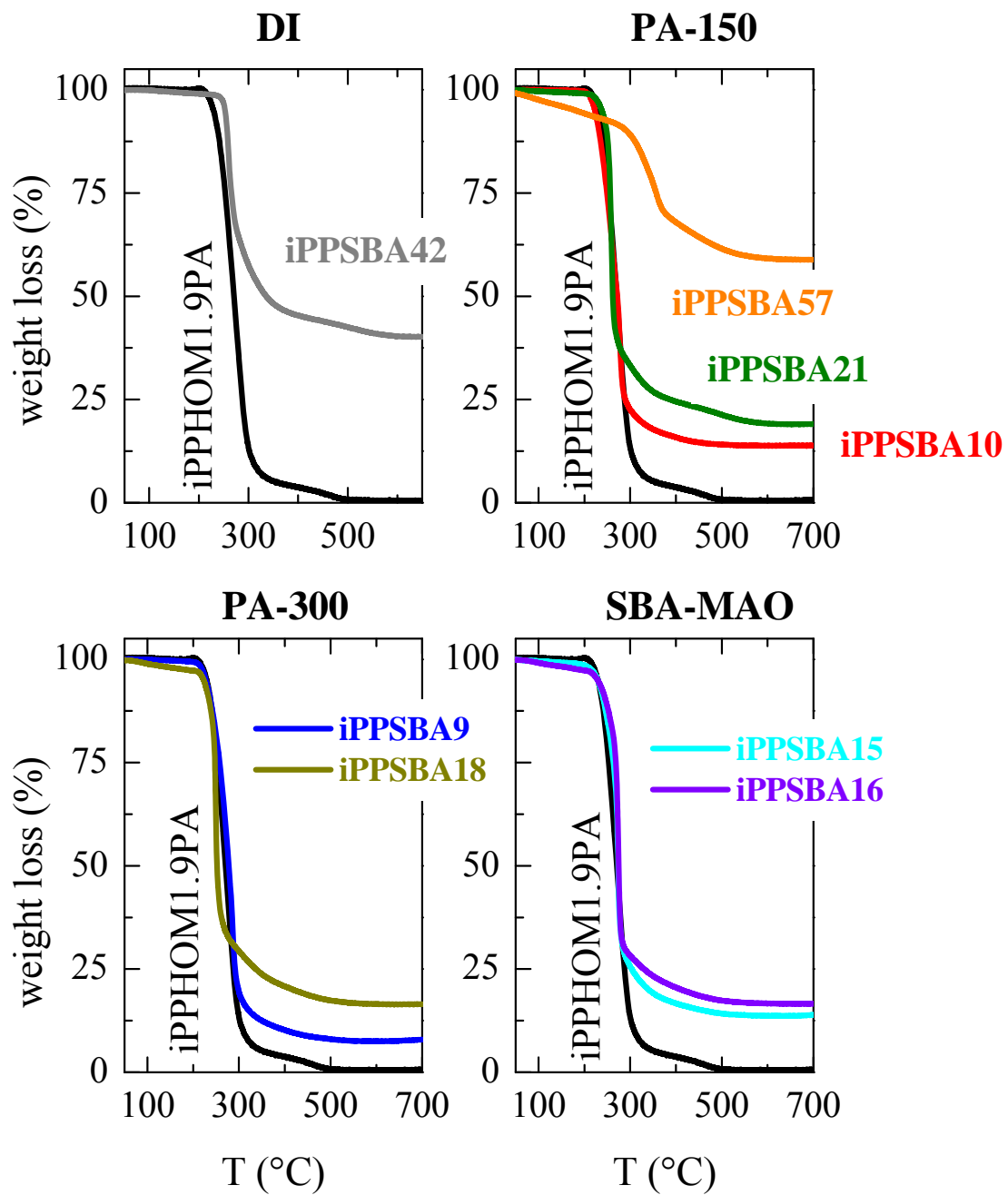

SBA-MAO

PA-150

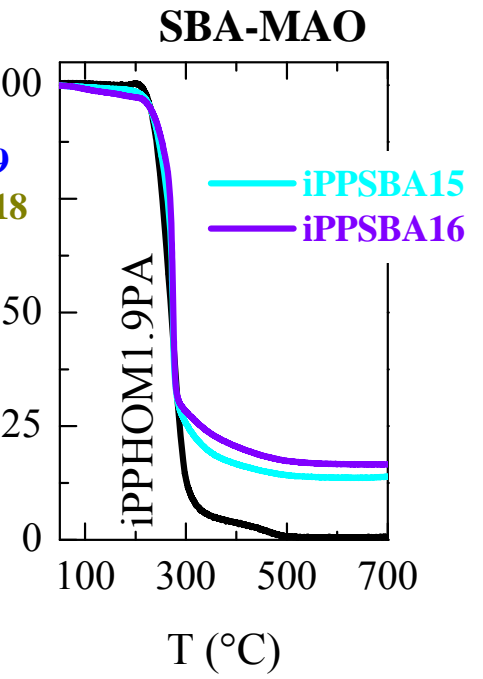

Figure 3. TGA curves under air atmosphere for the neat IPPHOM1.9PA (taken as reference) and its nanocomposites prepared by different catalyst impregnation approaches.

Figure 4 depicts the TGA derivatives from the curves in Figures 2 and 3 . They indicate that the presence of SBA-15 particles exert an influence more clearly observed than in the regular TGA curves, in relation to the ratio of different events. In the hybrids with the smallest particle amounts, the maximum is located at high temperatures while it is moved to lower temperatures as mesoporous silica content increases. Moreover, a narrowing of decomposition processes is observed as SBA-15 amount is raised. 
This behavior is somehow different to that found in composites of commercial iPP and SBA-15 particles prepared by melt extrusion, where a shift of the maximum to higher temperatures was observed compared with that exhibited by the homopolymer. It should be commented that the largest SBA-15 content used was $13 \mathrm{wt} . \%$ in those extruded materials [50].
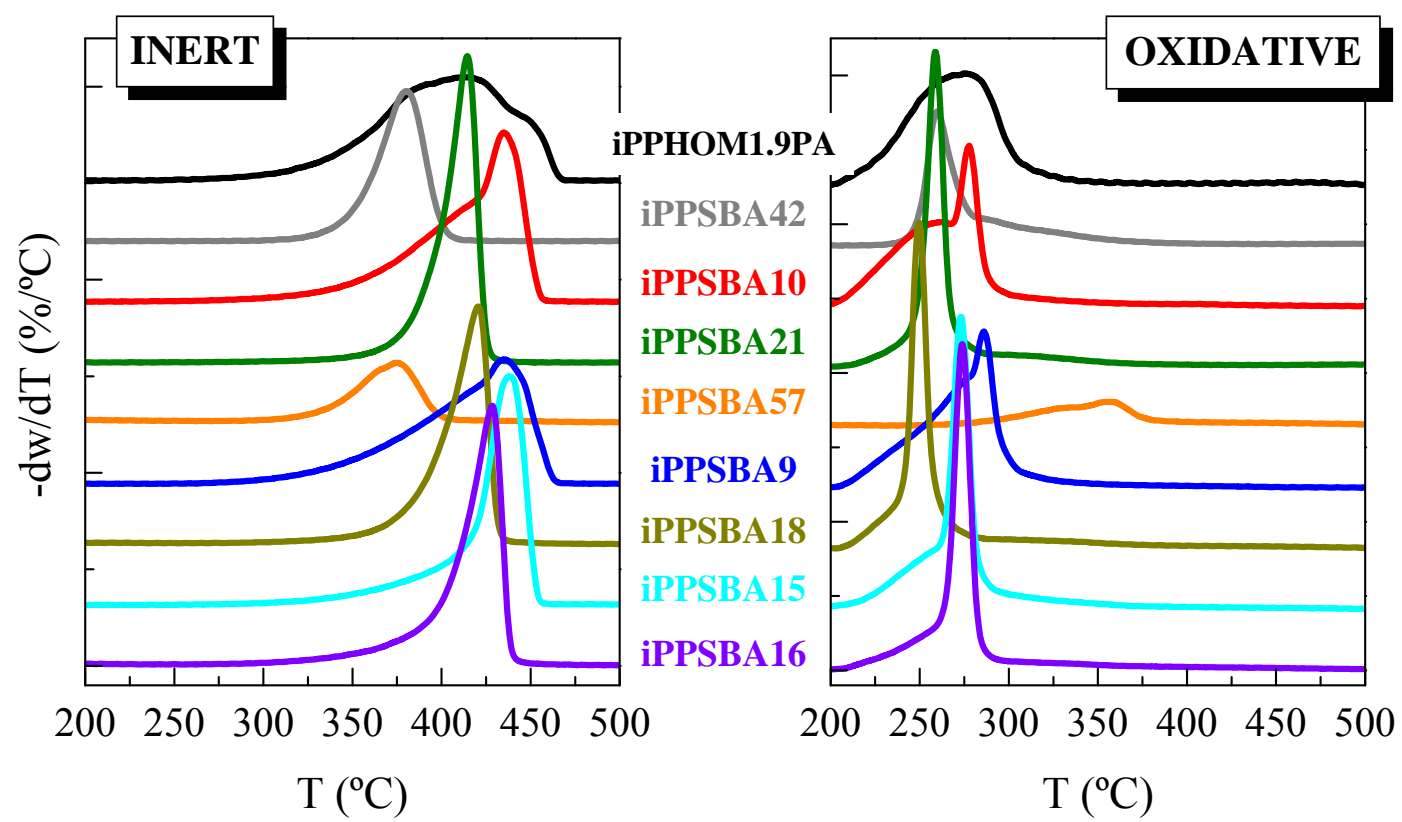

Figure 4. DTGA curves either under inert (left plot) or air (right plot) atmospheres for the neat iPPHOM1.9PA (taken as reference) and its nanocomposites prepared by different catalyst impregnation approaches.

\section{Phase transitions and structural features}

Figure 5 a shows the DSC endotherms obtained during the first melting process of some of the films, these selected nanocomposites covering the whole range of SBA-15 used. Two different endothermic events are observed within this temperature interval: that occurring at around $135^{\circ} \mathrm{C}$ and other process taking place close to $100{ }^{\circ} \mathrm{C}$. The former is ascribed to the melting of regular polypropylene chains. The melting temperature, $T_{m}$, value is relatively low (inferior to $140{ }^{\circ} \mathrm{C}$ ) because of the rather low isotacticity obtained in the resulting propylene materials, estimated by NMR and already commented, due to the metallocene catalyst employed. The endotherm at about $100{ }^{\circ} \mathrm{C}$ is also related to polypropylene crystallites but now, most probably, to those able to be developed within the nanometric SBA-15 channels. These geometrical constraints (pore diameter of these channels is around $6 \mathrm{~nm}$ [38]) are responsible that the iPP macrochains produced inside the SBA-15 mesoporosity during the synthetic procedure cannot grow as much as those iPP crystals located in the chains generated outside the SBA-15 pores. This feature was also found for hybrids based on HDPE and 
MCM-41, although melting of the confined polymer in those materials $[24,30,48]$ took place at around 80 ${ }^{\circ} \mathrm{C}$, the smaller pore size of those MCM-41 channels being one of the reasons for that temperature displacement. It was also observed at about $100{ }^{\circ} \mathrm{C}$ in nanocomposites of polyethylene with higher molecular masses $[32,49]$ and SBA-15.
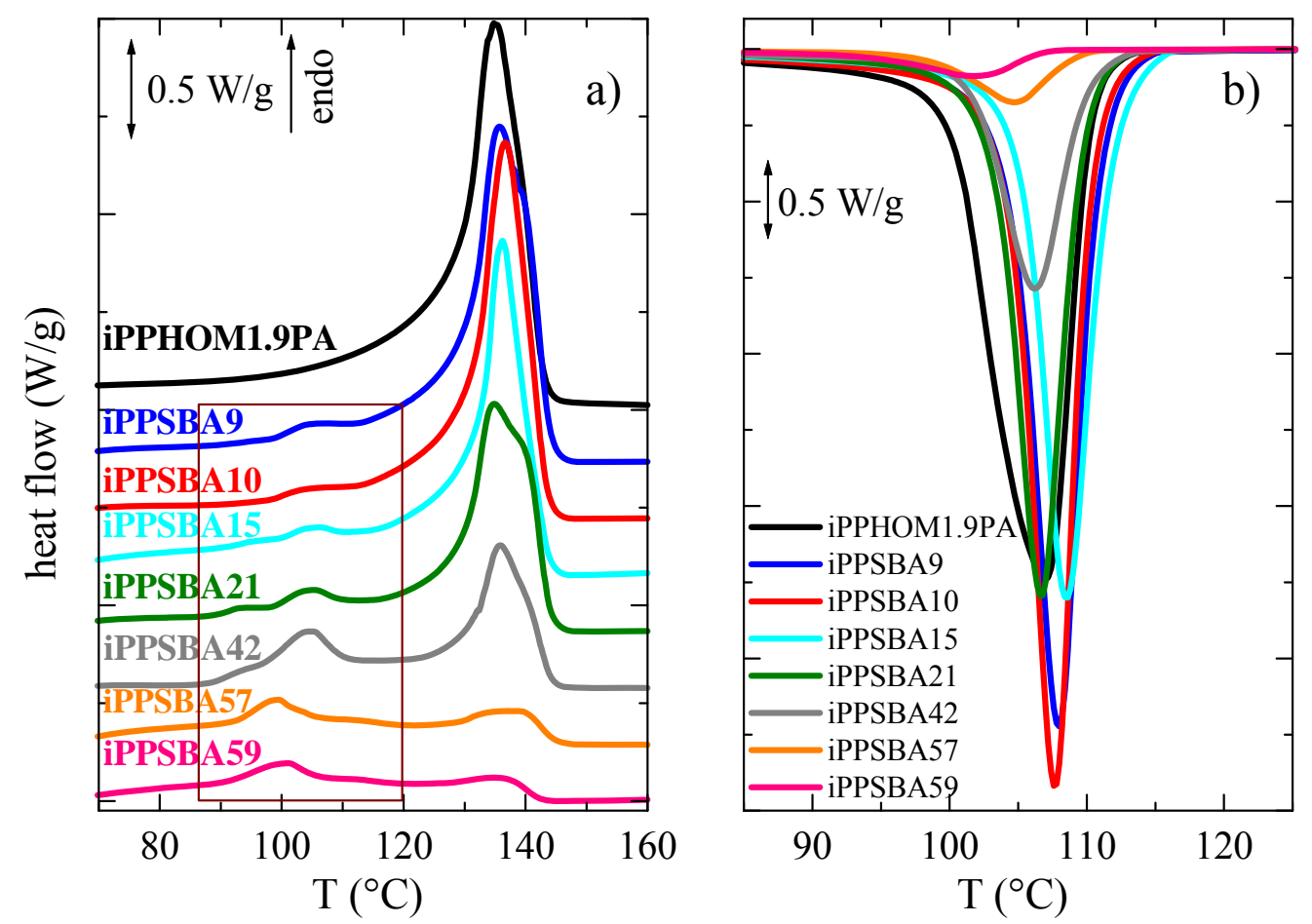

Figure 5. a) DSC endotherms related to the first melting run, shifted along $Y$ axis for a better visualization; b) DSC exotherms attained during crystallization process for the IPP homopolymer and different nanocomposites at several SBA-15 contents.

It can be also observed in Figure 5a that the total enthalpy deduced from these endotherms show a decreasing trend as SBA-15 content increases in the final material. The intensity of both endothermic peaks depends on the polypropylene existing at a given composite, as clearly deduced from Figure S4 of Supporting Information, where heat flow has been normalized to the actual amount of polypropylene at each specimen. After this normalization, a not very pronounced decrease of the overall polypropylene enthalpy is found up to around $40 \%$ of SBA-15, as depicted in Figure 6a. Nevertheless, the reduction in the total enthalpy becomes much steeper at higher SBA-15 contents. From these results, the values of the overall crystallinity, $f_{c}$ DSC, can be estimated. They are also detailed in Table 2 .

The enthalpy involved in the small endotherms, supposedly arising from the IPP crystallites confined inside the SBA-15 pores, is also shown in Figure 6a. An almost linear increase with the SBA- 
15 content is exhibited. Thus, rather significant amounts are obtained for high contents, in such a way that the relative value of that enthalpy in relation to the total enthalpy increases rather markedly, as shown in Figure 6b, showing values around 40\% for samples iPPSBA57 and iPPSBA59.

Table 2. SBA-15 wt. \% content calculated from TGA measurements under nitrogen and oxidative conditions, and average between them; melting $\left(T_{m}\right)$ and crystallization temperatures $\left(T_{c}\right)$, and overall crystallinity (normalized to the actual iPP content in the material) estimated by DSC $\left(f_{c}{ }^{D S C}\right)$ and WAXD $\left(\mathfrak{f}_{c}\right.$ WAXD) experiments.

\begin{tabular}{|c|c|c|c|c|c|c|c|c|}
\hline \multicolumn{2}{|c|}{ Sample } & $\begin{array}{c}\text { SBA-15inert } \\
\text { wt. } \%\end{array}$ & $\begin{array}{c}\text { SBA-15oxid. } \\
\text { wt. } \%\end{array}$ & $\begin{array}{c}\text { average } \\
\text { SBA-15 } \\
\text { wt. } \%\end{array}$ & $\begin{array}{c}\mathrm{T}_{\mathrm{m}} \\
\left({ }^{\circ} \mathrm{C}\right)\end{array}$ & $\begin{array}{c}\mathrm{T}_{\mathrm{c}} \\
\left({ }^{\circ} \mathrm{C}\right)\end{array}$ & $f_{C} \mathrm{DSC}$ & $f_{C}$ WAXD \\
\hline \multicolumn{2}{|c|}{ iPPHOM1.9PA } & 0 & 0 & 0 & 135.0 & 107.0 & 0.60 & 0.60 \\
\hline $\begin{array}{l}\text { iPPSBA42 } \\
\text { iPPSBA59 }\end{array}$ & $\bar{\Delta}$ & $\begin{array}{l}41 \\
59\end{array}$ & $\begin{array}{l}41 \\
59\end{array}$ & $\begin{array}{l}42 \\
59\end{array}$ & $\begin{array}{l}135.5 \\
135.0\end{array}$ & $\begin{array}{l}106.0 \\
102.0\end{array}$ & $\begin{array}{l}0.41 \\
0.20\end{array}$ & \\
\hline iPPSBA10 & \multirow{3}{*}{$\sum_{\frac{10}{1}}^{\frac{10}{2}}$} & 9 & 10 & 10 & 136.5 & 107.5 & 0.59 & 0.60 \\
\hline iPPSBA21 & & 21 & 21 & 21 & 135.0 & 106.5 & 0.53 & 0.50 \\
\hline iPPSBA57 & & 56 & 58 & 57 & 135.0 & 105.0 & 0.29 & 0.25 \\
\hline & \multirow{2}{*}{ 畩 } & 9 & 8 & 9 & 136.0 & 108.0 & 0.59 & \\
\hline iPPSBA18 & & 18 & 18 & 18 & 135.0 & 106.5 & 0.53 & \\
\hline iPPSBA15 & \multirow{2}{*}{ 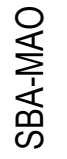 } & 15 & 16 & 15 & 136.0 & 108.5 & 0.55 & \\
\hline iPPSBA16 & & 16 & 16 & 16 & 136.0 & 107.0 & 0.56 & \\
\hline
\end{tabular}

Melting temperature of the main melting process does not change significantly with increasing the amount of SBA-15 particles (see Figures 5a and S5 of Supporting Information as well as the values in Table 2). On the other hand, both melting peaks become broader as SBA-15 content is raised in the final material. In fact, a bimodal endotherm is clearly seen in the melting process at about $100{ }^{\circ} \mathrm{C}$ for the nanocomposites with SBA-15 amount higher than 15 wt. \%, pointing out the presence of two crystallite populations with small size. 


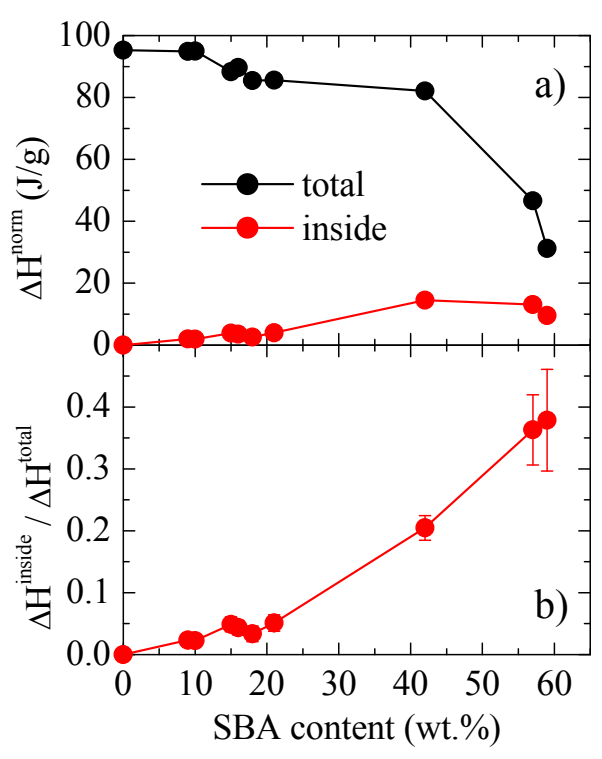

Figure 6. Variation with the SBA-15 content for the first melting in the different materials of: a) the total enthalpy and that inside the SBA-15 nanopores, after normalization to the actual polymer in the sample; and, b) of the corresponding relative enthalpy.

Figure $5 b$ displays the crystallization exotherms for the different hybrids. Two different trends can be observed, as deduced from data in Table 2 and Figure S5 in the Supporting Information. Relatively small amounts of SBA-15 particles, up to a $20 \mathrm{wt}$ \%, promote a small nucleating effect in the formation of polypropylene crystals, since crystallization temperature $\left(T_{c}\right)$ is moved to higher values. $A$ further increase of SBA-15 content leads to the occurrence of polypropylene crystallization at slightly lower temperatures at those high mesoporous amounts, i.e., presence of large amounts of mesoporous particles hinders the development of polypropylene crystallites.

Polypropylene is characterized for exhibiting a very interesting polymorphic behavior and different crystal lattices ( $\alpha, \beta, \gamma, \delta$ and a mesomorphic form) can be tuned through the temperature/ pressure/cooling rate imposed along processing together with the incorporation of specific nucleating additives and the variation of microstructural features [11,12,51-61]. Figure 7 shows the $X$ ray profiles, obtained with conventional radiation, for some of the nanocomposites.

An ordered array is observed in the angular interval ranging from 1 to $4^{\circ}$. This corresponds to the (110) and (200) reflections associated with the $p 6 \mathrm{~mm}$ hexagonal symmetry [62] of the periodically organized two-dimensional structures from the uniform mesopores existing in the SBA-15 particles. The first (100) order of this hexagonal arrangement is out of this $2 \theta$ scale since it appears at around $0.9^{\circ}$. It 
will be observed in the small angle X-ray diffraction profiles with synchrotron radiation, as discussed below. This hexagonal array is also seen in the different composites because the SBA-15 was used as a catalyst carrier during the polymerization stage and it was not removed at the end of that synthetic protocol. The results in Figure 7 (and those from SAXS, as seen below) indicate that the ordered SBA15 structure has been preserved in the resulting materials in spite of polypropylene chains are produced within the mesopores, as deduced from the presence of the endothermic peak at around $100{ }^{\circ} \mathrm{C}$.

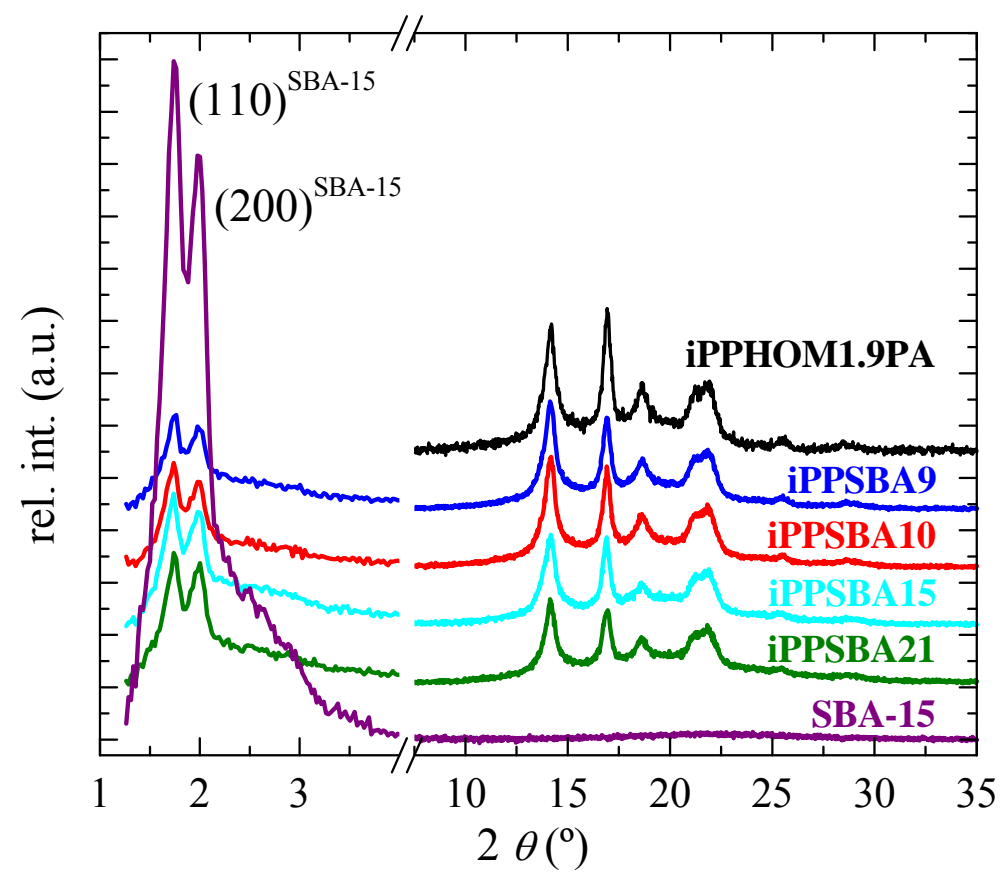

Figure 7. $\mathrm{X}$ ray diffraction patterns with conventional radiation at room temperature for the synthesized iPP homopolymer and different in situ nanocomposites with distinct SBA-15 content. The profile for neat SBA-15 is also shown.

At wide angle, it is clearly noticeable in Figure 7 that the polypropylene homopolymer shows the characteristics diffractions ascribed to its monoclinic a polymorph, as expected, since films have been processed by applying a relatively fast cooling from the molten state [63]. In spite of its low molar mass (as reported in Table 1), there is not evidence of any formation of the $\gamma$ lattice under these fast cooling conditions (although it is clearly observed for lower cooling rates, as seen below). This orthorhombic modification, easily identified because of its characteristic (117) diffraction peak at around $20^{\circ}$ in $2 \theta$ profiles [64], is specially favored in the case of iPP synthesized by metallocene catalysts due to the presence of errors homogeneously distributed among the different polymer chains [40]. Incorporation of SBA-15 does not affect the crystalline lattice that can be developed in the different nanocomposites 
under the imposed processing conditions and all of them show the common reflections from the monoclinic polymorph. No comparison can be established with previous results since the effect of SBA15 particles in the crystalline structure of polypropylene is not reported in literature.

In order to have a deeper understanding of the effect of SBA-15 in the crystalline structure of polypropylene and to analyze the first (100) order reflection of the hexagonal SBA-15 arrangement, realtime simultaneous small and wide angle $X$-ray experiments have been performed using synchrotron radiation in the homopolymer and in the samples synthesized by the PA-150 approach since they present a broad interval in SBA-15 particles content. As an example, Figure 8a shows the WAXD diffractograms for the first melting process of the rapidly cooled iPPSBA21 sample. As aforementioned, the monoclinic lattice of polypropylene was developed during processing and that crystalline structure is gaining perfection, as deduced from narrowing of reflections, by effect of temperature up to its melting process takes place, leading to an amorphous halo. Crystallization at $16{ }^{\circ} \mathrm{C} / \mathrm{min}$ was performed next (not shown), followed by the subsequent second melting (Figure 8b). The profile at $20^{\circ} \mathrm{C}$ in this second melting noticeably shows the appearance of the (117) reflection at around $2.25 \mathrm{~nm}^{-1}$ characteristic of the orthorhombic $\gamma$ polymorph. This finding is expected because the $\gamma$ lattice is favored at low crystallization rates in homopolymers $[40,63]$ and the second melting has been preceded by a relatively slow cooling at $16{ }^{\circ} \mathrm{C} / \mathrm{min}$. Incorporation of SBA-15 to polypropylene does not affect its capability of cocrystallizing both monoclinic and orthorhombic polymorphs if the rate used is appropriate. In addition, those $\gamma$ crystals melt, as usual, at temperatures lower than the monoclinic ones (see also in Figure 8b).

Figure $8 \mathrm{c}$ displays the corresponding WAXD 1D diffractograms at $20^{\circ} \mathrm{C}$ (first frame) presented for the homopolymer, composites iPPSBA10, iPPSBA21 and iPPSBA57 and neat SBA-15. It can be clearly observed that the diffractions, corresponding always to the monoclinic modification of iPP, are reduced very much in intensity as the SBA-15 content increases, similarly to the decrease found in the total enthalpy of melting depicted in Figure 6. Moreover, reflections become broader and less defined, indicating that the polypropylene crystals are smaller and less perfect as SBA-15 is raised. It can be also deduced from Figure $8 \mathrm{c}$ that SBA-15 is amorphous at short-range showing a wide halo, which is correspondingly reflected on the diffraction patterns of the nanocomposites, and especially in the case of iPPSBA57.

Crystallinity of polypropylene can be determined from these patterns. First of all, the corresponding amount of SBA-15 must be removed to obtain only the polymeric iPP component (as depicted in Figure S6 of Supporting Information). Once this is known, the amorphous iPP halo [41] at room temperature, which is derived from the amorphous profiles attained by variable-temperature $\mathrm{X}$-ray 
diffraction experiments after an appropriate shifting to account for the thermal expansion, is subtracted and values are achieved. Alternatively, the diffractogram of a totally amorphous elastomeric polypropylene can be considered as the amorphous polymeric component [65]. Very similar amorphous profiles are obtained in both two cases, and the corresponding degrees of crystallinity, $\mathrm{f}_{\mathrm{c}} \mathrm{WAXD}$, after normalization to the actual amount of polymer, are shown in the last column of Table 2 . These values are rather similar to those deduced from DSC results.
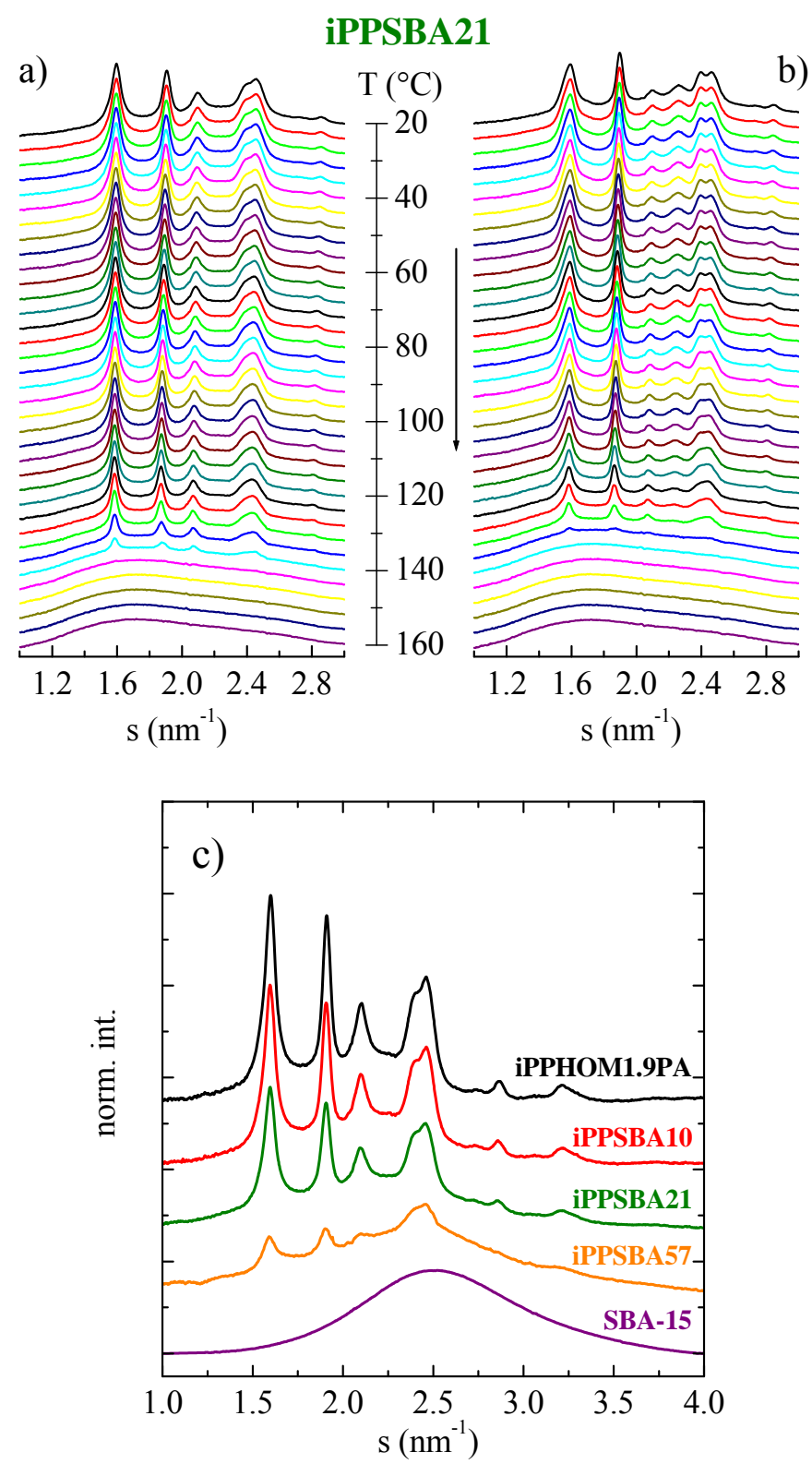

Figure 8. Real-time variable-temperature profiles at wide angle obtained with synchrotron radiation for:

a) the melting process at $16{ }^{\circ} \mathrm{C} / \mathrm{min}$ of the fast cooled iPPSBA21; and, b) its second melting after crystallization at $16{ }^{\circ} \mathrm{C} / \mathrm{min}$. c) Initial patterns at room temperature for homopolymer, SBA-15 and the hybrids prepared by the PA-150 approach. For clarity, only one every two frames is plotted in a) and b). 
Figure 9 shows the synchrotron SAXS 1D diffractograms for the melting of these three nanocomposites compared with the profiles exhibited by the neat SBA-15. The first order $(100)$ reflection characteristic from its hexagonal array is now clearly observed at around $s=0.110 \mathrm{~nm}^{-1}$ (distance between adjacent pores $=9.1 \mathrm{~nm}$ ). The vertical scales have been amplified for their better observation by the following factors: 8 times for iPPSBA-10, 4 times for iPPSBA-21 and 2 times for iPPSBA-57 in relation to the pristine mesoporous silica.

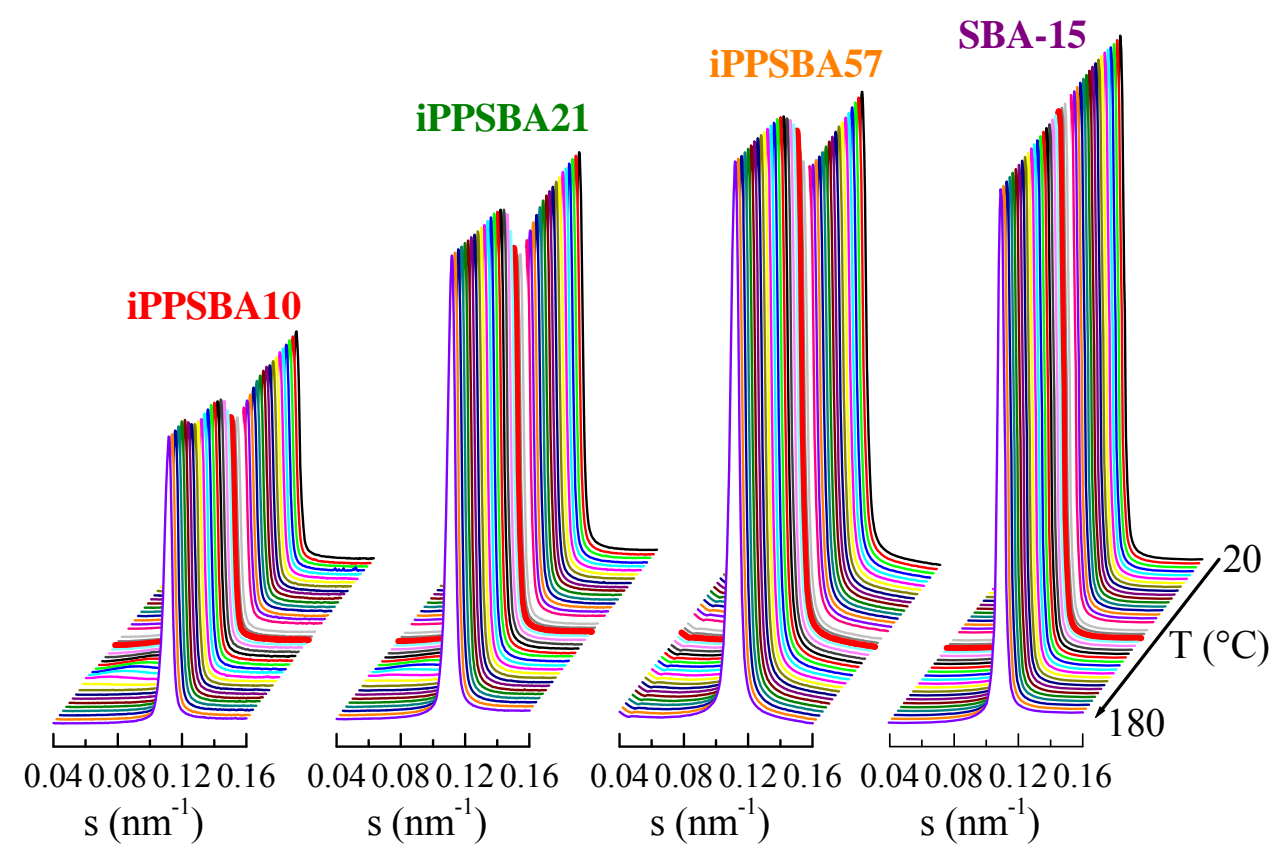

Figure 9. Synchrotron SAXS 1D diffractograms for the melting at $16{ }^{\circ} \mathrm{C} / \mathrm{min}$ of the indicated samples.

For clarity, only one every two frames is plotted.

Position of the (100) SBA-15 reflection remains practically unchanged, independently of presence and amount of polypropylene in the different materials. Its area, however, undergoes a clear and systematic increase, dependent on SBA-15 content, in all the iPP nanocomposites in the interval from around 90 to $120{ }^{\circ} \mathrm{C}$, as observed in Figure 9, where the frames at $\mathrm{T}=100{ }^{\circ} \mathrm{C}$ have been highlighted in red. This frame appears at the beginning of the upward step for samples iPPSBA10 and iPPSBA21, but it is at the middle of the rising step for iPPSBA57. That is in perfect agreement with the thermal results depicted in Figure 5. Interestingly, that increase is not observed for neat SBA-15, which shows a practically constant intensity in the temperature interval of interest, since no significant variation takes place.

Previous results on different SBA-15 composites [66-68] suggested that the intensity of the SBA-15 diffraction is dependent on the degree of pore filling and also on the scattering contrast 
eventually existing between walls and inside of the SBA-15 channels. Consequently, the variations in the intensity of the SBA-15 peak are most probably an indirect proof of the polymer chains being arranged inside the SBA-15 pores. The corresponding confined crystals, with very low values in their lateral size, adjusted to the pore diameter, shall melt at temperatures considerably lower than those for the crystallites outside the channels, as deduced by applying an appropriate version of the GibbsThomson equation $[69,70]$.

The intensity changes in Figure 9 for the SBA-15 (100) diffraction have been quantified as function of temperature. As an example, Figure 10a shows its variation with temperature for composite iPPSBA57. The corresponding derivative of that variation is represented in Figure 10b, compared with its DSC curve in the region of the low-temperature endotherms. An excellent agreement is observed between these two techniques, especially when considering that the DSC results have been performed at $20^{\circ} \mathrm{C} / \mathrm{min}$ while that rate is $16^{\circ} \mathrm{C} / \mathrm{min}$ for the synchrotron experiments.

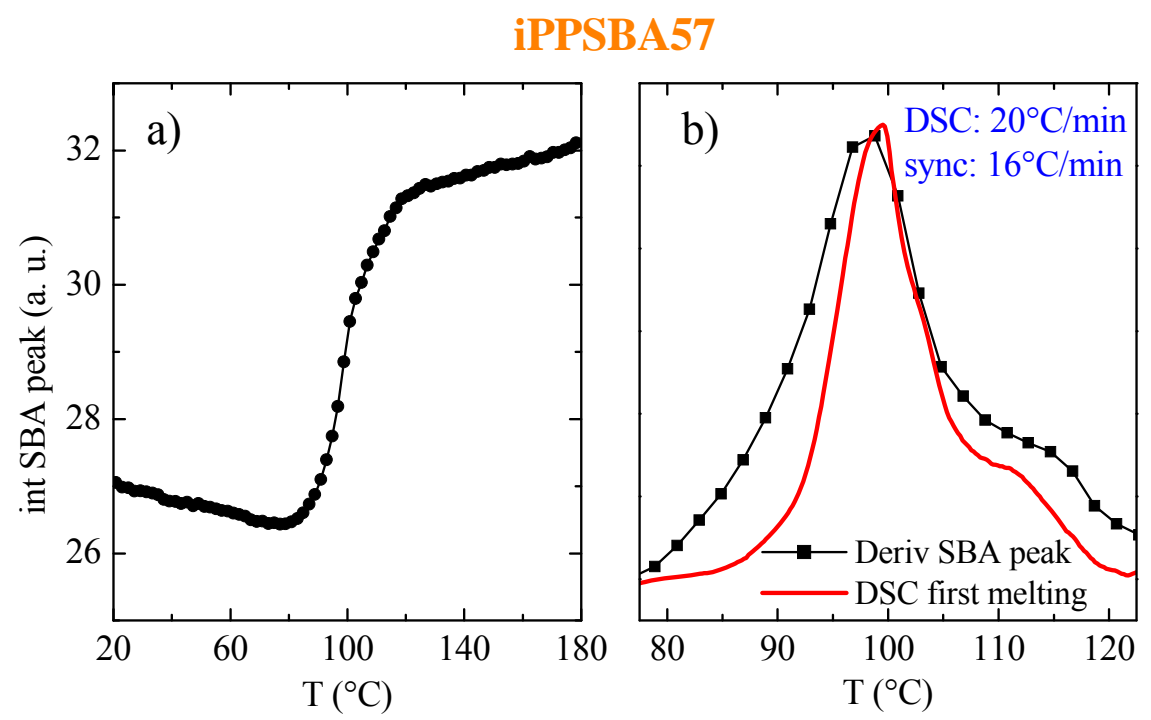

Figure 10. a) Variation with temperature of the intensity of the SBA-15 (100) diffraction for the composite iPPSBA57. b) Corresponding derivative of that variation, compared with its DSC curve in the region of the low-temperature endotherms.

In situ polymerization can lead to final materials with much higher compositions in SBA-15 compared with those able to be reached by melt extrusion and these physical aspects can be easily evaluated. As previously reported for polyethylene $[30,48]$, the interpretation of the DSC results represented in Figure 5a, which is now corroborated by SAXS measurements, indicates the occurrence of confinement effects in some polypropylene macrochains, characterized by a substantial lower melting 
temperature because of their smaller size, as commented above. This change on the SBA-15 SAXS intensity during heating is, then, associated with the variation that the interior of SBA-15 pores undergo in electronic density through the melting of those confined crystals to an isotropic state.

On the other hand, the regular polypropylene crystallites developed in the chains polymerized outside of the SBA-15 channels show long spacings at smaller $s$ values than the main SBA-15 diffraction, as firstly deduced from Figure 9 . That is more clearly shown in Figure 11, where the Lorentzcorrected SAXS profiles for iPPHOM1.9PA homopolymer and for the iPPSBA21 nanocomposite are presented.
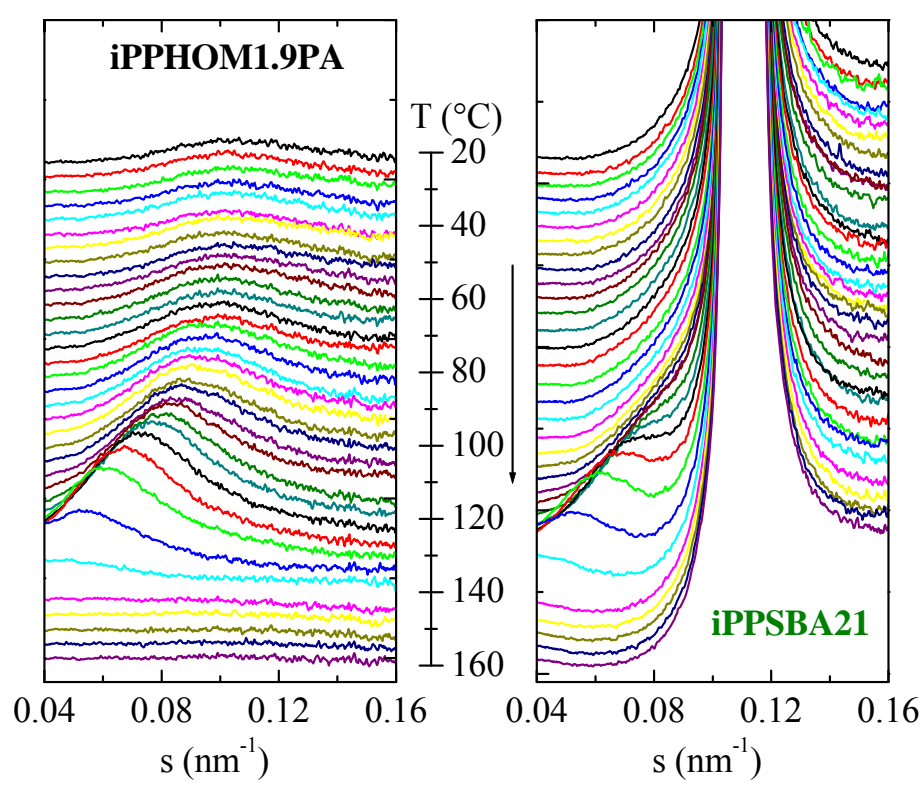

Figure 11. Real-time variable-temperature Lorentz-corrected SAXS profiles obtained at $16^{\circ} \mathrm{C} / \mathrm{min}$ with synchrotron radiation for iPPHOM1.9PA homopolymer (left plot) and for the iPPSBA21 nanocomposite (right plot). Only one every two frames is plotted for clarity.

It can be observed that the homopolymer exhibits a clear long spacing at room temperature, at $s=0.106 \mathrm{~nm}^{-1}, L=9.43 \mathrm{~nm}$. An important shift to lower $s$ values of the peak related to this long spacing is observed at increasing temperatures, associated with the improvement of the iPP crystalline morphology by effect of temperature and, then, the thickening of its crystallites. Long spacing is a magnitude used in the lamellar stack model theory of semicrystalline polymers that is directly related to the electron density differences between the two phases (amorphous and crystalline), so that it undergoes rather important variations during crystallization, recrystallization or melting processes in semicrystalline polymers [71-74]. Data prove that most probable long spacing remains initially rather 
constant, up to around $80^{\circ} \mathrm{C}$, where an increase in intensity and shift to lower $s$ values is observed, which becomes more important above $110^{\circ} \mathrm{C}$, followed by a complete diminishment and disappearance above around $140{ }^{\circ} \mathrm{C}$, related to the melting process of those crystallites existing in the external macrochains.

An important problem, related to the presence of SBA-15, occurs for the nanocomposites. The expected iPP long spacing will appear at around $s=0.106 \mathrm{~nm}^{-1}$ but the main diffraction of SBA-15 is seen at around $s=0.110 \mathrm{~nm}^{-1}$. Consequently, the SBA-15 reflection will overlap with the IPP long spacing, as observed in the right plot of Figure 11. Only at temperatures above around $110^{\circ} \mathrm{C}$, when the significant improvement by effect of temperature of the iPP crystals takes place, the long spacing is observed, although also partially overlapped with the tail of the SBA-15 peak. This superposition precludes a correct determination of the iPP long spacing in the nanocomposites. Nevertheless, it can be deduced from Figure 11 that the values of the long spacing are rather similar to those of the homopolymer in the region with extensive thickening of the iPP crystals.

Another parameter extracted from the SAXS curves is the relative invariant [71-73] directly associated with the scattering power, which can be determined from the area under the SAXS curve. In the present case, that determination has been performed from the SAXS results up to a value of $s=$ $0.09 \mathrm{~nm}^{-1}$, so that the main SBA-15 peak is not taken into account. The results are shown in Figure 12. Regarding the homopolymer, its relative invariant exhibits a significant increase coinciding with the region of crystal thickening, falling down sharply during its final melting.

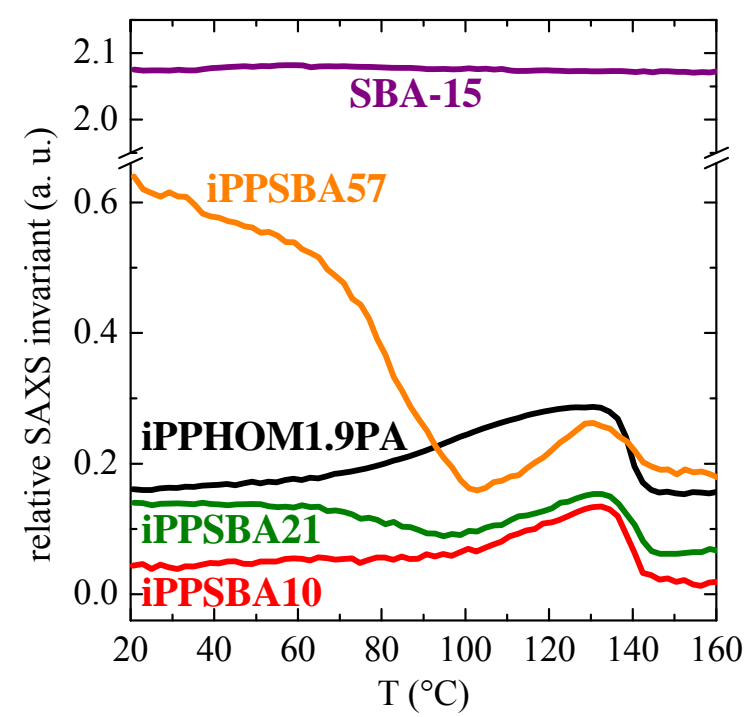

Figure 12. Variation with temperature of the relative SAXS invariant for the homopolymer, the nanocomposites prepared by the PA150 approach, and the neat SBA-15. 
A rather analogous behavior is observed for specimen iPPSBA10, although now the relative invariant is considerably smaller, and a subtle discontinuity in the increase due to crystal thickening appears in the temperature interval around $90-100^{\circ} \mathrm{C}$. When the SBA-15 content is increased, sample iPPSBA21, a clear minimum at around $100^{\circ} \mathrm{C}$ is now exhibited, which is much more pronounced for the nanocomposite iPPSBA57. The invariant for the neat SBA-15 is, however, practically constant with temperature, and its value is much higher than the ones for the other samples. Two conclusions can be extracted from all these results. First of all, the relative invariant is dominated in the nanocomposites by the scattering power of the SBA-15 particles in the region of the lower $s$ values observed. Secondly, this relative invariant is again rather sensitive to the melting, at around $100{ }^{\circ} \mathrm{C}$, of the crystallites confined in the SBA-15 channels.

\section{Mechanical response by indentation measurements}

Mesoporous SBA-15 particles have been used, at first, as catalyst carrier. Nevertheless, they were left within the resultant materials once polymerization was finished in order to act also during their shelf life as reinforcements. Evaluation of the mechanical performance of some of these nanocomposites turns out of great interest. Figure 13 shows the results derived from indentation measurements.
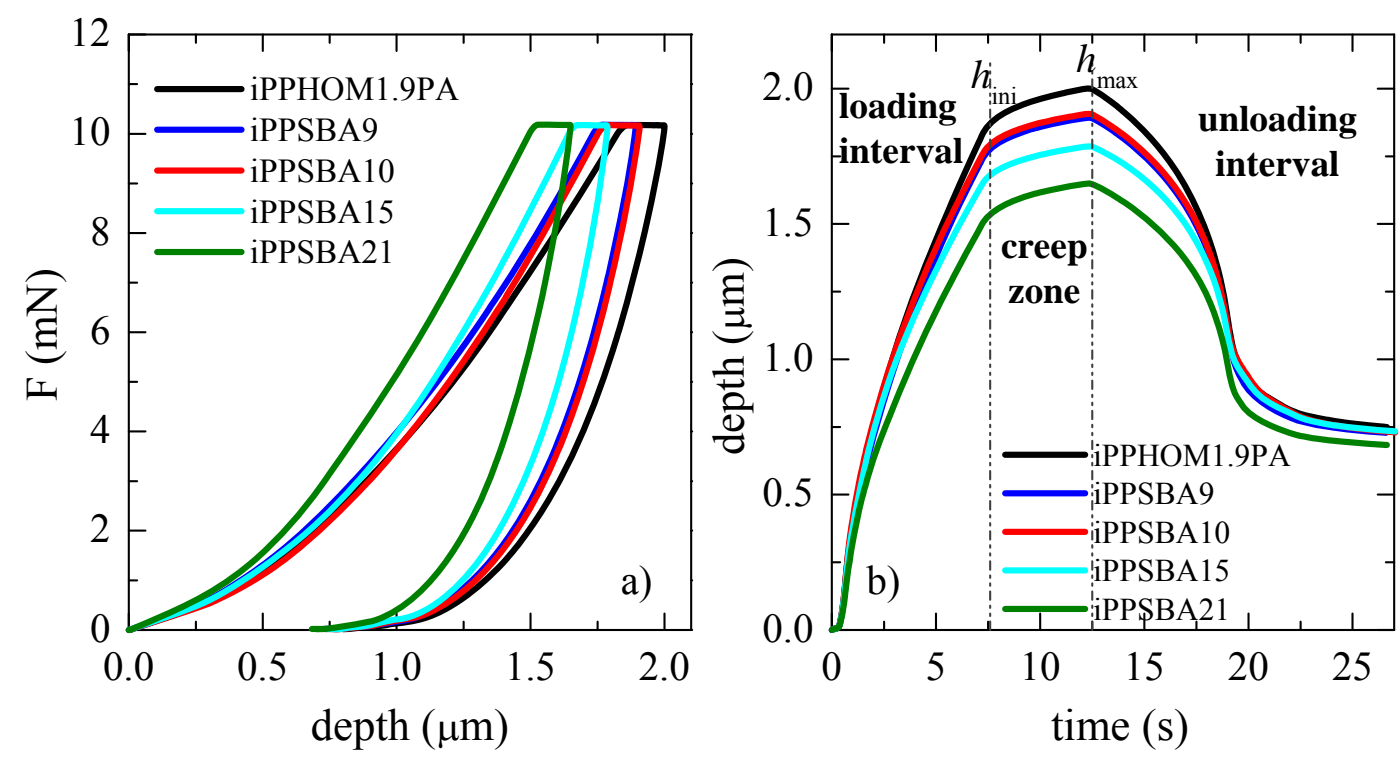

Figure 13. a) Load-maintenance-unload vs. depth curves; and, b) indenter depth dependence on experimental time for the iPPHOM1.9PA homopolymer and some of the nanocomposites with different SBA-15 contents. 
Figure 13a depicts the influence of SBA-15 particles in the capability of indenter to penetrate in the surface of different materials. The depth reached is reduced, at a given load, as SBA-15 content increases. It is clearly seen that differences between IPPSBA9 and IPPSBA10 are within the experimental error, indicating that the most important variable is the amount of SBA-15 and not the synthetic approach used. These results are related to the stiffness characteristics, either in the bulk or in the surface, as shown in the Figure $14 a$ where modulus, $E_{i t}$, and hardness, $H_{i t}$, of indentation, respectively, have been represented. It is noticeable that the effect of SBA-15 is in the bulk greater than in the surface.

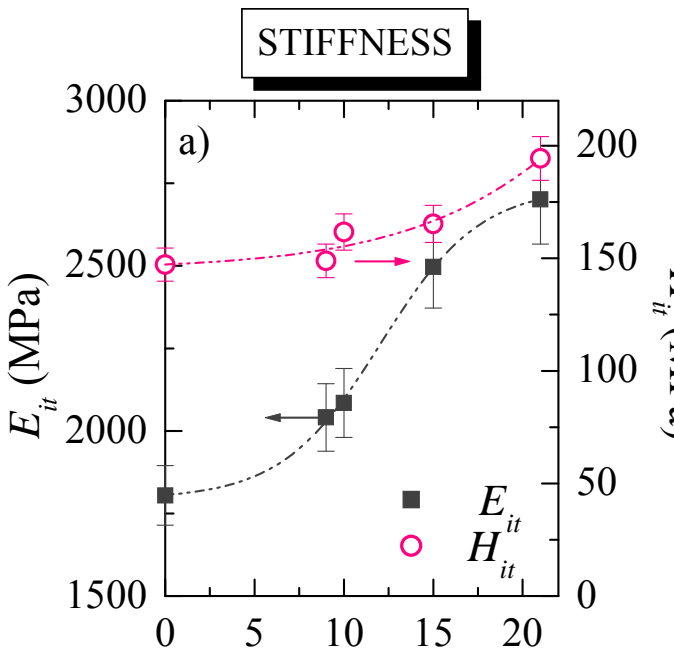

SBA-15 content (wt.\%)

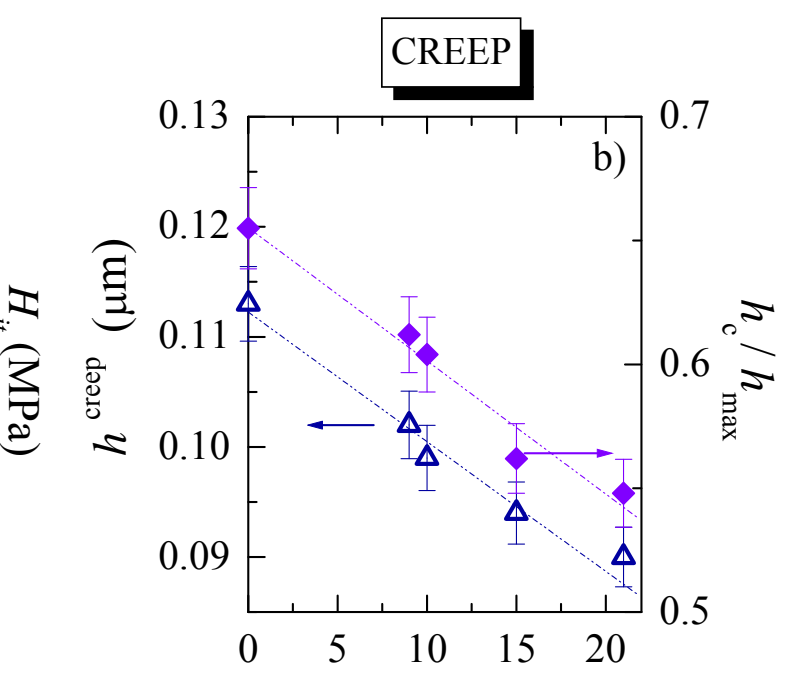

SBA-15 content (wt. \%)

Figure 14. Mechanical parameters determined from indentation measurements for the IPPHOM1.9PA homopolymer and some of the nanocomposites with different SBA-15 contents: a) related to stiffness: modulus, $E_{i t}$ (left axis scale), and hardness, $H_{i t}$ (right axis scale). And, b) related to creep: $h$ creep, which is defined as: $h^{\text {creep }}=h_{\max }-h_{\text {ini, }}$ i.e., the difference of depth at the end and at the beginning of the process at constant load (left); and ratio between the $h_{\mathrm{c}}$ (contact depth) and maximum depth (right).

Figure $13 \mathrm{~b}$ allows focusing the attention in the creep region at a constant load of $10 \mathrm{mN}$. The indenter in the homopolymer is able to reach a higher depth than those achieved in the several nanocomposites. Moreover, this depth is dependent on SBA-15 content. This feature is clearly observed in Figure 14b where the $h$ creep, i.e., the change of indentation depth along creep stage defined as the

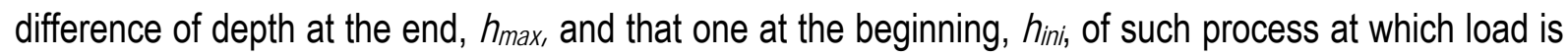
maintained constant, and the $h_{\mathrm{c}} / h_{\max }$ ratio (where $h_{\mathrm{c}}$ is the indentation depth in contact with indenter [75]) decrease rather linearly with SBA-15 content. Since both parameters are associated with the 
dimensional stability of the ultimate materials, it is concluded that this magnitude becomes improved by the presence of these mesoporous particles. Their influence is, then, very important because their incorporation overcomes the reduction observed in the polypropylene crystallinity as their content is raised. Accordingly, maintenance of mesoporous particles after polymerization leads to a positive mechanical effect since they exert a significant reinforcing role and contribute to enlarge the final dimensional stability.

\section{Conclusions}

New materials based on commodity polypropylene and SBA-15 microparticles have been prepared by in situ polymerization. The mesoporous SBA-15 has been used as catalyst carrier and as reinforcement. Immobilization of the catalytic system has been performed through several approaches and the pre-activation strategy has led to the better productivity results. Resultant dispersion of SBA-15 in the polypropylene appears rather uniform and formation of aggregates of large size is not detected. Moreover, nanocomposites maintain the features in long-range ordering of the mesoporous silica, independently of the preparation approach, indicating that the polypropylene macrochains do not significantly alter the ordered arrangement of the SBA-15 mesostructure.

Observation of a secondary endothermic event in the nanocomposites at around $100{ }^{\circ} \mathrm{C}$ points out the presence of crystallites with significantly smaller size together with those melting at about $140^{\circ} \mathrm{C}$. This endotherm has been ascribed to the melting of the iPP crystals existing within SBA-15 pores and channels and the enthalpy involved increases with the SBA-15 content.

The main (100) SBA-15 reflection in the SAXS region turns out a decisive sensor of presence of iPP crystallites within this mesostructure. Its intensity exhibits an important change at around $100{ }^{\circ} \mathrm{C}$. This variation has been quantified as function of temperature and its corresponding derivative correlates very well with the DSC curve in the region of the low-temperature endotherm. Moreover, the long spacing of iPP appears rather overlapped with that main SBA-15 diffraction in the nanocomposites. This superposition precludes its accurate determination at low temperatures, although rather similar values to those from the homopolymer are found in the temperature interval involving the extensive thickening of the iPP crystals. Variation with temperature of the SAXS invariant shows in the nanocomposites that such invariant is dominated in this region by the scattering power of the SBA-15 particles, becoming rather sensitive to the melting at around $100{ }^{\circ} \mathrm{C}$ of the crystallites confined in the SBA- 15 channels.

Ultimate mechanical performance, as deduced from indentation measurements, improves with SBA-15 incorporation (without varying the final processing temperature). Thus, stiffness increases and 
deformability is reduced in the nanocomposites as SBA-15 content rises and polypropylene amount within channels is also enlarged. It can be concluded that the most important variable is the amount of SBA-15 and not the synthetic approach used.

\section{Acknowledgements}

The financial support from project MAT2016-79869-C2-1-P (AEI/FEDER, UE) is greatly acknowledged. Ms. R Barranco-García also thanks her pre-doctoral funding (BES-2014-070972) associated with the MINECO (MAT2013-47972-C2-1-P) project. The synchrotron experiments were performed at beamline BL11-NCD at ALBA Synchrotron Light Facility with the collaboration of ALBA staff. Authors are grateful for the funding received.

\section{References}

\footnotetext{
1 X. Liu, G. Zheng, K. Dai, Z. Jia, S. Li, Ch. Liu, J. Chen, Ch. Shen, Q. Li, J Mater Sci 46 (2011) 7830-7838.

2 Y. Pan, X. Liu, S. Shi, Ch. Liu, K. Dai, R. Yin, D.W. Schubert, G. Zheng, Ch. Shen, Macromol. Mater. Eng. 301 (2016) 1468-1472.

3 Y. Pan, X. Guo, G. Zheng, Ch. Liu, Q. Chen, Ch. Shen, X. Liu, Macromol. Mater. Eng. 303 (2018) 1800083.

4 J. Jiang, X. Liu, M. Lian, Y. Pan, Q. Chen, H. Liu, G. Zheng, Z. Guo, D.W. Schubert, Ch. Shen, Ch. Liu, Polym. Testing 67 (2018) 183-189.

${ }^{5}$ X. Wang, Y. Pan, Y. Qin, M. Voigt, X. Liu, G. Zheng, Q. Chen, D.W. Schubert, Ch. Liu, Ch. Shen, Polym. Testing 69 (2018) 478-484.

6 W. Kaminsky, Catalysis Today 20 (1994) 257-271.

7 J. Arranz-Andrés, J.L. Guevara, T. Velilla, R. Quijada, R. Benavente, E. Pérez, M.L. Cerrada, Polymer 46 (2005) 1228712297.

${ }^{8}$ H. Palza, J.M. López-Majada, R. Quijada, J.M. Pereña, R. Benavente, E. Pérez, M.L.Cerrada, Macromol. Chem. Phys. 209 (2008) 2259-2267.

${ }_{9}^{9}$ M.L. Cerrada, M.J. Polo-Corpa, R. Benavente, E. Pérez, T. Velilla, R. Quijada, Macromolecules 42 (2009) 702-708.

10 M.J. Polo-Corpa, R. Benavente, T. Velilla, R. Quijada, E. Pérez, M.L. Cerrada, Eur. Polym. J. 46 (2010) 1345-1354.

11 E. Pérez, J.M. Gómez-Elvira, R. Benavente, M.L. Cerrada, Macromolecules 45 (2012) 6481-6490.

12 J. Arranz-Andrés, R. Parrilla, M.L. Cerrada, E. Pérez. Macromolecules 46 (2013) 8557-8568.

13 K. Soga, H. J. Kim, T. Shiono, Macromol. Chem. Phys. 195 (1994) 3347-3360.

14 M.C. Sacchi, D. Zucchi, I. Tritto, P.Locatelli, T. Dallocco, Macromol. Rapid Comm. 16 (1995) 581-590.

15 N. Semikolenova, V.A.Zakharov, Macromol. Chem. Phys. 198 (1997) 2889-2897.

16 S.C. Moreira, M.F.V. Marques, Eur. Polym. J. 37 (2001) 2123-2130.

17 Y. Huang, Y. Qin, Y. Zhou, H. Niu, Z.-Z. Yu, J.-Y. Dong, Chem. Mater. 22 (2010) 4096-4102.

18 N. Wang, Y. Qin, Y. Huang, H. Niu, J.-Y. Dong, Y. Wang, Applied Catalysis A: General 435- 436 (2012) 107- 114.

19 M.A. Milani, D. Gonzalez, R. Quijada, N.R.S. Basso, A.P. Graebin, G.B. Galland, Polym. Sci. Part B: Polym. Phys. 50 (2012) 3598-3605.

${ }^{20}$ M.A. Milani, D. Gonzalez, R. Quijada, N.R.S. Basso, M.L. Cerrada, D.S. Azambuja, G.B. Galland, Compos. Sci. Technol. $84(2013) 1-7$.

21 J.S. Beck, J.C. Vartuli, W.J. Roth, M.E. Leonowicz, C.T. Kresge, K.D. Schmitt, C.T.-W. Chu, D.H. Olson, E.W. Sheppard, S.B. McCullen, J.B. Higgins, J.L. Schlenkert, J. Am. Chem. Soc. 114 (1992) 10834-10843.

22 D.Y. Zhao, J.L. Feng, Q.S. Huo, N. Melosh, G.H. Fredrickson, B.F. Chmelka, G.D. Stucky, Science 279 (1998) 548-552.

${ }^{23}$ M.L. Cerrada, E. Pérez, J.P. Lourenço, J.M. Campos, M. Rosário Ribeiro, Micropor. Mesopor. Mat. 130 (2010) 215-223.

${ }^{24}$ A. Kurek, S. Mark, M. Enders, M.O. Kristen, R. Mülhaupt, Macromol. Rapid Comm. 31 (2010) 1359-1363.

25 J.M. Campos, J.P. Lourenço, H. Cramail, M.R. Ribeiro, Prog. Polym. Sci. 37 (2012) 1764-1804.

26 X.C. Dong, L, Wang, G.H. Jiang, Z.R. Zhao, T.X. Sun, H.J. Yu, W.Q. Wang, J. Mol. Catal. A-Chem. 240 (2005) $239-244$.

27 G.C. Xu, A.Y. Li, L. De Zhang, X.Y. Yu, T. Xie, G.S. Wu, J. Reinforced Plastics and Composites, 23 (2004) 1365-1372.

28 J.M. Campos, M.R. Ribeiro, J.P. Lourenço, A. Fernandes, J. Mol. Catal. A-Chem. 277 (2007) 93-101.

29 J.M. Campos, J.P. Lourenço, E. Pérez, M.L. Cerrada, M.R. Ribeiro, J. Nanosci. Nanotechn. 9 (2009) 3966-3974.
} 
${ }^{30}$ M.L. Cerrada, E. Pérez, J.P. Lourenço, A. Bento, M.R. Ribeiro, Polymer 54 (2013) 2611-2620.

${ }^{31}$ A. Bento, J.P. Lourenço, A. Fernandes, M.L. Cerrada, M.R. Ribeiro, ChemCatChem 5 (2013) 966-976.

32 A.E. Ferreira, M.L. Cerrada, E. Pérez, V. Lorenzo, H. Cramail, J.P. Lourenço, M.R. Ribeiro. Micropor. Mesopor. Mat. 232 (2016) 13-25.

${ }^{33}$ A.E. Ferreira, M.L. Cerrada, E. Pérez V. Lorenzo, E. Vallés, J. Ressia, H. Cramail, J.P. Lourenço, M.R. Ribeiro, eXPRESS Polym. Lett. 11 (2017) 344-361.

34 M.F.V. Marques, C.C. Pombo, R.A. Silva, A. Conte, Eur. Polym. J. 39 (2003) 561-567.

35 V.I. Costa Vayá, P.G. Belelli, J.H.Z. dos Santos, M.L. Ferreira, D.E. Damiani, J. Catal. 204 (2001) 1-10.

${ }^{36}$ K.-T. Li, F.-S. Ko, J. Appl. Polym. Sci. 107 (2008) 1387-1394.

${ }^{37}$ D.M González, R. Quijada, M. Yazdani-Pedram, J.P. Lourenço, M.R. Ribeiro, Polym. Int. 65 (2016) 320-326.

${ }^{38}$ A. Deryło-Marczewska, M. Zienkiewicz-Strzałka, K. Skrzypczyńska, Andrzej Światkowski, Krzysztof Kuśmierek, Adsorption 22 (2016) 801-812.

39 E.B. Bond, J.E. Spruiell, J.S. Lin, J. Polym. Sci. Part B: Polym. Phys. 37 (1999) 3050-3064.

40 R. Krache, R. Benavente, J.M. López-Majada, J.M. Pereña, M.L. Cerrada, E. Pérez, Macromolecules 40 (2007) 6871-6878.

${ }^{41}$ E. Pérez, M.L. Cerrada, R. Benavente, J.M. Gómez-Elvira. Macromol. Res. 19 (2011) 1179-1185.

42 W.C. Oliver, G.M. Pharr, J. Mater. Res. 7 (1992) 1564-1583.

${ }^{43}$ M. Hoyos, P. Tiemblo, J.M. Gómez-Elvira, Polymer 48 (2007) 183-194.

${ }^{44}$ H.-W. Wong, L.J. Broadbelt, Ind. Eng. Chem. Res. 40 (2001) 4716-4723.

${ }^{45}$ L. Achimsky, L. Audouin, J. Verdu, J. Rychly, L. Matisova-Rychla, Polym. Degrad. Stab. 58 (1997) 283-289.

${ }^{46}$ H. Nakatani, S. Suzuki, T. Tanaka, M. Terano, Polymer 46 (2005) 12366-12371.

47 A. Bento, J. P. Lourenço, A. Fernandes, M.R. Ribeiro, J. Arranz-Andrés, V. Lorenzo, M.L. Cerrada, J. Membr. Sci. 415-416 (2012) 702-711.

${ }^{48}$ M.L. Cerrada, A. Bento, E. Pérez, V. Lorenzo, J.P. Lourenço, M.R. Ribeiro, Micropor. Mesopor. Mat. 232 (2016) 86-96.

${ }^{49}$ A.E. Ferreira, M.L. Cerrada, E. Pérez, V. Lorenzo, H. Cramail, J.P. Lourenço, R. Quijada, M.R. Ribeiro, Eur. Polym. J. 85 (2016) 298-312.

${ }^{50}$ R. Barranco-García, M.L. Cerrada, J.A. Ressia, E.M. Vallés, A. García-Peñas, E. Pérez, J.M. Gómez-Elvira, Polym. Degrad. Stab. (doi: 10.1016/j.polymdegradstab.2018.06.006).

51 G. Natta; P. Corradini, Nuovo Cimento Suppl. 15 (1960) 40-51.

52 A. Turner-Jones, J.M. Aizlewood, D.R. Beckett, Makromol. Chem. 75 (1964) 134-158.

53 S. Brückner, S.V. Meille, V. Petraccone, B. Pirozzi, Prog. Polym. Sci. 16 (1991) 361-404.

54J.J. Varga, Macromol. Sci., Phys. B 41 (2002) 1121-1171.

55 I.L. Hosier, R.G. Alamo, P. Esteso, J.R. Isasi, L. Mandelkern, Macromolecules 36 (2003) 5623-5636.

${ }_{56}$ B. Poon, M. Rogunova, A. Hiltner, E. Baer, S.P. Chum, A. Galeski. E, Piorkowska, Macromolecules 38 (2005) 1232-1243.

${ }^{57}$ C. De Rosa, S. Dello lacono, F. Auriemma, E. Ciaccia, L. Resconi, Macromolecules 39 (2006) 6098-6109.

${ }^{58}$ C. De Rosa, O.R. Ballesteros, F. Auriemma, M.R. Di Caprio, Macromolecules 45 (2012) 2749-2763.

59 L. Boragno, P. Stagnaro, F. Forlini, F. Azzurri, G.C. Alfonso, Polymer 54 (2013) 1656-1662.

60 A. García-Peñas, J.M. Gómez-Elvira, R. Barranco-García, E. Pérez, M.L. Cerrada, Polymer 99 (2016) 112-121.

${ }^{61}$ A.García-Peñas, J.M. Gómez-Elvira, V. Lorenzo, E. Pérez, M.L. Cerrada, Polymer 130 (2017) 17-25.

62 D. Zhao, Q. Huo, J. Feng, B.F. Chmelka, G.D. Stucky, J. Am. Chem. Soc. 120 (1998) 6024-6036.

63 J. Arranz-Andrés, B. Peña, R. Benavente, E. Pérez, M. L. Cerrada, Eur. Polym. J. 43 (2007) 2357-2370.

${ }^{64}$ A. Turner-Jones, Polymer 12 (1971) 487-508.

65 S. Mansel, E. Pérez, R. Benavente, J.M. Pereña, A. Bello, W. Röll, R. Kirsten, S. Beck, H.-H. Brintzinger, Macromol. Chem. Phys. 200 (1999) 1292-1297.

${ }^{66}$ X.Wang, X. Ma, Ch. Song, D.R. Locke, S. Siefert, R.E. Winans, J.Möllmer, M. Lange, A. Möller, R. Gläser, Micropor. Mesopor. Mat. 169 (2013) 103-111.

67 J. Sauer, F. Marlow, F. Schüth, Phys. Chem. Chem. Phys. 3 (2001) 5579-5584.

${ }^{68}$ W. Hammond, E. Prouzet, S.D. Mahanti, T.J. Pinnavaia, Micropor. Mesopor. Mat. 27 (1999) 19-25.

69 U.W. Gedde, Polymer Physics, Chapman \& Hall, London, 1995.

70 K. Shin, E. Woo, Y.G. Jeong, C. Kim, J. Huh, K.-W. Kim, Macromolecules 40 (2007) 6617-6623.

${ }^{71}$ F.J. Baltá-Calleja, C.G.Vonk, X-Ray Scattering of Synthetic Polymers. Amsterdam, Elservier 1989.

${ }^{72}$ A.J. Ryan, J.L. Stanford, W. Bras, T.M.W. Nye, Polymer 38 (1997) 759-768.

${ }^{73}$ B. Crist, J. Polym. Sci., Part B: Polym. Phys. 39 (2001) 2454-2460.

74 J.L. de la Fuente, M. Wilhelm, H.W. Spiess, E.L. Madruga, M. Fernández Garcia, M.L. Cerrada, Polymer 46 (2005) 4544-4553.

75 S.T. Cholake, M.R. Mada, R. Kumar, P. Boughton, S. Bandyopadhyay, Ann. Materials Sci. Eng. 2 (2015) id1022. 